\title{
A nonparametric test for the comparison of first-order structures of spatial point processes
}

\author{
I. Fuentes-Santos ${ }^{\mathrm{a}, *}$, W. González-Manteiga ${ }^{\mathrm{b}}$, J. Mateu $^{\mathrm{c}}$ \\ ${ }^{a}$ Marine Research Institute. Spanish National Research Council, Vigo, Spain \\ ${ }^{b}$ Faculty of Mathematics, University of Santiago de Compostela, Spain \\ ${ }^{c}$ Department of Mathematics, University Jaume I, Castellón, Spain
}

\begin{abstract}
Comparing the spatial distribution of two spatial point patterns is an important issue in many scientific areas such as ecology, epidemiology or environmental risk assessment. However, up to date, the analysis of multitype point processes has been mainly focused on searching for interactions between events of different patterns, i.e. on the second-order structure, while the first-order structure has received less attention. This work proposes testing the similarity between two spatial point patterns through the comparison of their densities of event locations. For this purpose, we consider the usual squared discrepancy measure to propose a nonparametric statistical test. The asymptotic normal distribution of the associated statistic provides a calibration procedure. The simulation study conducted to analyze the performance of the test shows that this calibration can be too conservative and supports the use of a proposed bootstrap calibration. The performance of the test is also illustrated throughout its application to the analysis of the spatial patterns of wildfires registered in Galicia (NW Spain) during 2006.
\end{abstract}

Keywords: bootstrap calibration, first-order intensity, inhomogeneous Poisson point process, kernel smoothing, wildfires

\section{Introduction}

A common question in the analysis of multitype spatial point processes is whether two types of events have the same spatial structure. This ques-

\footnotetext{
*Corresponding author: isafusa@gmail.com (I. Fuentes-Santos)
} 
tion can arise in different scientific contexts such as ecology, where we can compare the spatial distribution of several species in a given region, in the analysis of environmental risks, where we can wonder if the spatial distribution of arson and natural wildfires is the same, or in epidemiology, where the spatial patterns of disease cases are compared with the population at risk. However, up to date the analysis of multitype point processes has been mainly focused on testing for interactions between spatial patterns through Monte Carlo tests based on second-order characteristics such as the K-cross and the mark correlation functions (Diggle, 2013; Ripley, 1981). For instance, these techniques have been widely used to search for relationships between different types of wildfires (Fuentes-Santos et al., 2013; Hering et al., 2009; Juan et al., 2012), or to analyze species distribution in plant ecology (Fibich et al., 2016; Getzin et al., 2008; Perry et al., 2006).

A spatial point process is a stochastic process governing the location of a random number of events, $\mathbf{X}=\left\{\mathbf{x}_{\mathbf{1}}, \ldots, \mathbf{x}_{\mathbf{N}}\right\}$, irregularly placed in a planar region $W \subset \mathbb{R}^{2}$. If each event has associated any measure or mark, we have a marked point process. A multitype point process is a marked point process with categorical marks that define different groups or types of events (Diggle, 2013). Throughout this paper, point processes and patterns are denoted in bold capitals, and events are denoted in bold. The firstorder intensity function (Diggle, 2013; Illian et al., 2008), which measures the expected number of events per unit area, is defined as follows

$$
\lambda(x)=\lim _{|d x| \rightarrow \infty}\left\{\frac{E[N(d x)]}{|d x|}\right\}
$$

where $|d x|$ and $N(d x)$ denote the area and the number of events of $\mathbf{X}$ in $d x$. The first-order intensity function is crucial to describe the spatial distribution of events in a spatial point process, in particular, $\lambda(\cdot)$ characterizes the distribution of spatial Poisson point processes. The dependence structure of univariate and multitype point processes is characterized by second-order functions such as the K-function $K(r)$, which measures the expected number of further events within distance $r$ of an arbitrary event in a given point process (Ripley, 1977), and the K-cross function $K_{i j}(r)$, which measures the expected number of type $j$ events within distance $r$ of an arbitrary type $i$ event (Ripley, 1981). 
Two spatial point patterns with the same spatial structure can be seen as the type $i$ and type $j$ patterns of a random labeled bivariate point process, or as independent realizations of a point process, in both cases their K-functions are equal, $K_{i}(r)=K_{j}(r)$ (Diggle, 2013; Ripley, 1977). This property suggests using nonparametric tests based on these second-oder characteristics to check the similarity between two observed patterns (Hahn, 2012). However, differences between the K-functions of two inhomogeneous spatial point patterns can reflect differences in the first-order intensities or in the dependence structure. On the other hand, recent studies have introduced area-based tests (Alba-Fernández et al., 2016; Andresen, 2009), which count the number of events within predefined spatial units (e.g. quadrants, space-filling curves) to measure local and global discrepancies between two observed point patterns.

A natural way to test whether two spatial point patterns have the same spatial structure should be through comparison of first-order properties, since these properties describe the spatial distribution of events in the observation domain. In this line, Kelsall and Diggle (1995) proposed a kernel estimator for relative risk functions, defined as the ratio between the first-order intensities of disease cases and a control population. They also considered associated surface p-values of tolerance contours based on Monte Carlo simulations of the null hypothesis of equal spatial distribution of disease cases and controls, including a global Monte Carlo homogeneity test. Davies and Hazelton (2010) introduced an adaptive kernel estimator of the relative risk function, which is asymptotically normal, and provides tolerance contours without the need of Monte Carlo simulations.

The main aim of this work is to develop a new nonparametric procedure to test whether two spatial point patterns have the same first-order structure. For this purpose, we take into account that the first-order intensity functions of spatial point patterns with the same structure are proportional, and consequently, they have the same density of event locations (Cucala, 2006). The equivalence between the density of event locations of spatial Poisson point processes and the bivariate density function of random variables, and the consistency of the kernel estimator of the density of event locations (Cucala, 2006; Fuentes-Santos et al., 2016), suggest to extend the test introduced by Duong et al. (2012) for comparison of multivariate distributions to the point process framework. 
The plan of this paper is the following. In Section 2 we introduce the statistical test, obtain its asymptotic null distribution to calibrate the distribution of the associated statistic, and propose an alternative calibration by bootstrap. The performance of the test is scrutinized by a simulation study in Section 3 and through its application to the analysis of wildfires in Galicia (NW Spain) in Section 4. The paper ends with some conclusions in Section 5. Appendix A provides the proof of Theorem 1, which establishes the asymptotic null distribution of the test statistic.

\section{Comparison between kernel intensity estimators}

\subsection{The statistical test}

Let $\mathbf{X}=\left\{\mathbf{x}_{\mathbf{1}}, \ldots, \mathbf{x}_{\mathbf{N}}\right\}$ be a realization of a bivariate inhomogeneous spatial Poisson point process observed in a bounded region $W \subset \mathbb{R}^{2}$, and $\mathbf{X}_{1}=\left\{\mathbf{x}_{1}, \ldots, \mathbf{x}_{\mathbf{N}_{1}}\right\}=\left\{\mathbf{x}_{1,1}, \ldots, \mathbf{x}_{1, \mathbf{N}_{1}}\right\}, \mathbf{X}_{2}=\left\{\mathbf{x}_{\mathbf{N}_{1}+\mathbf{1}}, \ldots, \mathbf{x}_{\mathbf{N}_{1}+\mathbf{N}_{2}}\right\}=$ $\left\{\mathbf{x}_{2,1}, \ldots, \mathbf{x}_{\mathbf{2}, \mathbf{N}_{\mathbf{2}}}\right\}$, where $N=N_{1}+N_{2}$, the spatial patterns of type 1 and type 2 events in $\mathbf{X}$. We denote by $\lambda_{1}(x)$ and $\lambda_{2}(x)$ the first-order intensities of $\mathbf{X}_{1}$ and $\mathbf{X}_{2}$, and by $\lambda_{01}(x)=\lambda_{1}(x) / m_{1}, \lambda_{02}(x)=\lambda_{2}(x) / m_{2}$ their densities of event locations (Cucala, 2006), where $m_{1}$ and $m_{2}$ are the expected number of events of each point process.

The density of event locations for $\mathbf{X}_{j}, j=1,2$ can be estimated by kernel smoothing

$$
\begin{aligned}
\hat{\lambda}_{0 j, G_{j}}(x) & =\left(p_{G_{j}}(x) N_{j}\right)^{-1} \sum_{i=1}^{N_{j}} k_{G_{j}}\left(x-\mathbf{x}_{\mathbf{j}, \mathbf{i}}\right) I\left[N_{j} \neq 0\right] \\
& =\left(p_{G_{j}}(x) N_{j}\right)^{-1}\left|G_{j}\right|^{-1 / 2} \sum_{i=1}^{N_{j}} k\left(G_{j}^{-1 / 2}\left(x-\mathbf{x}_{\mathbf{j}, \mathbf{i}}\right)\right) I\left[N_{j} \neq 0\right]
\end{aligned}
$$

where the kernel function, $k(\cdot)$, is a radially symmetric bivariate probability density function, $G_{j}, j=1,2$, is the symmetric and positive-definite bandwidth matrix, $\left|G_{j}\right|$ denotes the determinant of $G_{j}$, and $p_{G_{j}}(x)=\int_{W} k_{G_{j}}(x-y) d y$ is the edge-correction term.

The Poisson assumption is needed to guarantee the consistency of the kernel estimator of the densities of event locations (Fuentes-Santos et al., 2016) and to obtain the asymptotic null distribution of the test statistic that 
will be later introduced. However the nonparametric test proposed in this work can be applied to non-Poisson point processes. Notice that we can not distinguish between hetereogeneity and interaction in an observed point pattern unless we have some additional information, such as the knowledge of covariates or the assumption of a parametric model (Diggle, 2013). Thus, a common practice in the analysis of spatial point patterns is assuming that the point process is Poisson, estimating the first-order intensity, and then estimating the second-order properties to test the Poisson assumption.

If $\mathbf{X}_{1}$ and $\mathbf{X}_{2}$ have the same spatial distribution, their densities of event locations are equal. On the other hand, conditional to the number of events $N_{j}=n_{j}$, the observed patterns can be seen as random samples of the bivariate random distributions with densities $\lambda_{0 j}(\cdot), j=1,2$. Thus, we can extend the proposal of Duong et al. (2012) for multivariate data to the spatial point process framework and use a squared discrepancy measure to test the null hypothesis $\mathcal{H}_{0}: \lambda_{01}(x)=\lambda_{02}(x)=\lambda_{0}(x)$ almost $\forall x \in W$, of the form

$$
\begin{aligned}
T & =\int_{W}\left(\lambda_{01}(x)-\lambda_{02}(x)\right)^{2} d x \\
& =\int_{W} \lambda_{01}(x)^{2} d x+\int_{W} \lambda_{02}(x)^{2} d x-\int_{W} \lambda_{01}(x) \lambda_{02}(x) d x-\int_{W} \lambda_{02}(x) \lambda_{01}(x) d x \\
& =\mathbb{E}_{\mathbf{X}_{\mathbf{1}}}\left[\lambda_{01}(x)\right]+\mathbb{E}_{\mathbf{X}_{\mathbf{2}}}\left[\lambda_{02}(x)\right]-\mathbb{E}_{\mathbf{X}_{\mathbf{2}}}\left[\lambda_{01}(x)\right]-\mathbb{E}_{\mathbf{X}_{\mathbf{1}}}\left[\lambda_{02}(x)\right]
\end{aligned}
$$

Expression (2) can be rewritten as $T=\psi_{1}+\psi_{2}-\left(\psi_{12}+\psi_{21}\right)$, where $\psi_{j}=$ $\mathbb{E}_{\mathbf{X}_{\mathbf{j}}}\left[\lambda_{0 j}(x)\right]$ and $\psi_{i j}=\mathbb{E}_{\mathbf{X}_{\mathbf{i}}}\left[\lambda_{0 j}(x)\right]$, for $i, j=1,2$. Assuming $W=\mathbb{R}^{2}$ to avoid the limitation of edge-effects, and using kernel smoothing to estimate each component of $T$, we obtain the test statistic

$$
\hat{T}=\hat{\psi}_{1}+\hat{\psi}_{2}-\left(\hat{\psi}_{12}+\hat{\psi}_{21}\right)
$$


where,

$$
\begin{aligned}
& \hat{\psi}_{1}=N_{1}^{-2} \sum_{i_{1}=1}^{N_{1}} \sum_{i_{2}=1}^{N_{1}} k_{G_{1}}\left(\mathbf{x}_{\mathbf{i}_{\mathbf{1}}}-\mathbf{x}_{\mathbf{i}_{\mathbf{2}}}\right) I\left(N_{1}>0\right) \\
& \hat{\psi}_{2}=N_{2}^{-2} \sum_{j_{1}=N_{1}+1}^{N} \sum_{j_{2}=N_{1}+1}^{N} k_{G_{2}}\left(\mathbf{x}_{\mathbf{j}_{1}}-\mathbf{x}_{\mathbf{j}_{2}}\right) I\left(N_{2}>0\right) \\
& \hat{\psi}_{12}=\left(N_{1} N_{2}\right)^{-1} \sum_{i=1}^{N_{1}} \sum_{j=N_{1}+1}^{N} k_{G_{1}}\left(\mathbf{x}_{\mathbf{i}}-\mathbf{x}_{\mathbf{j}}\right) I\left(N_{1}>0\right) I\left(N_{2}>0\right) \\
& \hat{\psi}_{21}=\left(N_{1} N_{2}\right)^{-1} \sum_{i=1}^{N_{1}} \sum_{j=N_{1}+1}^{N} k_{G_{2}}\left(\mathbf{x}_{\mathbf{i}}-\mathbf{x}_{\mathbf{j}}\right) I\left(N_{1}>0\right) I\left(N_{2}>0\right)
\end{aligned}
$$

and where $G_{1}$ and $G_{2}$ are the bandwidth matrices for the kernel estimators of the density functionals $\psi_{1}$ and $\psi_{2}$.

\subsection{Asymptotic theory and calibration}

In the multivariate density framework, Duong et al. (2012) showed that the null distribution of $\hat{T}$ is asymptotically normal, and proposed nonparametric estimates of its mean and variance to calibrate the test. The following theorem establishes the asymptotic null distribution of $\hat{T}$ in our framework of spatial point processes.

Theorem 1. Given a bivariate inhomogeneous Poisson point pattern, $\mathbf{X}=$ $\left\{\mathbf{x}_{1}, \ldots, \mathbf{x}_{\mathbf{N}}\right\}$, let $\mathbf{X}_{\mathbf{1}}=\left\{\mathbf{x}_{\mathbf{1}}, \ldots, \mathbf{x}_{\mathbf{N}_{1}}\right\}, \mathbf{X}_{\mathbf{2}}=\left\{\mathbf{x}_{\mathbf{N}_{1}+\mathbf{1}}, \ldots, \mathbf{x}_{\mathbf{N}_{1}+\mathbf{N}_{2}}\right\}$, with $N=N_{1}+N_{2}$, be the spatial patterns of type 1 and type 2 events in $\mathbf{X}$, and $\lambda_{01}(x)=\lambda_{1}(x) / m_{1}, \lambda_{02}(x)=\lambda_{2}(x) / m_{2}$ their densities of event locations. Under the following regularity conditions for $j=1,2$

H1 The densities of event locations $\lambda_{0 j}(\cdot)$ have partial derivatives up to order 2, all their second-order partial derivatives are bounded, continuous and square integrable.

H2 The bandwidth matrices $G_{j}$ used to compute $\hat{T}$ are symmetric and positive-definite, and such that all entries of $G_{j}$ tend to 0 and $m_{j}\left|G_{j}\right|^{1 / 2} \rightarrow$ $\infty$ as $m_{j} \rightarrow \infty$. 
H3 The kernel function $k(\cdot)$ is a continuous, symmetric, square integrable density function such that $\int_{\mathbb{R}^{2}} u u^{T} k(u) d u=\mu_{2}(k) I_{2}$, where $I_{2}$ is the two-dimensional identity matrix and $0<\mu_{2}(k)<\infty$.

and assuming that the null hypothesis $\mathcal{H}_{0}: \lambda_{01}(x)=\lambda_{02}(x)=\lambda_{0}(x)$ for all $x \in W$ holds, then

$$
\hat{Z}=\frac{\hat{T}-\mu_{\hat{T}}}{\sigma_{\hat{T}}} \stackrel{d}{\rightarrow} N(0,1)
$$

where

$$
\mu_{\hat{T}}=\mathbb{E} \hat{T}=\left(A\left(m_{1}\right)\left|G_{1}\right|^{-1 / 2}+A\left(m_{2}\right)\left|G_{2}\right|^{-1 / 2}\right) k(0)
$$

and

$$
\sigma_{\hat{T}}^{2}=\mathbb{V a r} \hat{T}=3\left(A\left(m_{1}\right)+A\left(m_{2}\right)\right)\left(\int \lambda_{0}^{3}(x) d x-\psi^{2}\right)(1+o(1))
$$

where $A\left(m_{j}\right)=\mathbb{E}\left[\frac{1}{N_{j}} I\left(N_{j}>0\right)\right]$, and $\psi=\int \lambda_{0}^{2}(x) d x$.

See the proof in Appendix A.

To use the asymptotic normal distribution of $\hat{T}$ given by expression (4) to calibrate our test, we first need to estimate $\mu_{\hat{T}}$ and $\sigma_{\hat{T}}$. Given that for any observed patterns the number of events $N_{j}=n_{j}, j=1,2$ is fixed, and consequently $\mathbf{X}_{\mathbf{j}}$ can be seen as a random sample of the bivariate variable with density $\lambda_{0 j}(\cdot)$, we can use the nonparametric estimators introduced by Duong et al. (2012), where $A\left(m_{j}\right)$ in expression (5) is estimated by $1 / n_{j}$ in our case.

Theorem 1 provides an asymptotic calibration for our test. However, this asymptotic normal calibration can produce a test that does not achieve the nominal significance level, specially when we compare small point patterns. For this reason, we propose using smooth bootstrap to generate resamples of $\mathbf{X}_{1}$ and $\mathbf{X}_{\mathbf{2}}$ under $\mathcal{H}_{0}$ and estimate the null distribution of $\hat{T}$. The bootstrap calibration can be implemented as follows:

1. Compute the test statistic $\hat{T}$ for the observed patterns, $\mathbf{X}_{\mathbf{1}}$ and $\mathbf{X}_{\mathbf{2}}$.

2. Let $\mathbf{X}=\left\{\mathbf{X}_{\mathbf{1}}, \mathbf{X}_{\mathbf{2}}\right\}$ be the unmarked spatial point pattern comprising both types of events, obtain the kernel estimator of its density of event locations, $\hat{\lambda}_{0, H}(x)$. 
3. For $b=1, \ldots, B$ :

3.1 Generate a bivariate spatial point process $\mathbf{X}_{\mathbf{b}}^{*}=\left\{\mathbf{X}_{\mathbf{1}, \mathbf{b}}^{*}, \mathbf{X}_{\mathbf{2}, \mathbf{b}}^{*}\right\}$ where for $j=1,2, \mathbf{X}_{\mathbf{j}, \mathbf{b}}^{*}$ are realizations of spatial Poisson point processes with first-order intensities $n_{j} \hat{\lambda}_{0, H}(x)$, being $n_{j}$ the number of events in $\mathbf{X}_{\mathbf{j}}$.

3.2 Compute the corresponding test statistic $\hat{T}_{b}^{*}$.

4. Given a significance level $\alpha$, the critical point is the $100(1-\alpha)$-percentile of $\hat{T}_{b}^{*}, b=1, \ldots, B, \hat{T}_{100(1-\alpha)}^{*}$. Thus we reject the null hypothesis when $\hat{T}>\hat{T}_{100(1-\alpha)}^{*}$.

Two bandwidth selection procedures are involved in this bootstrap algorithm. The bandwidth matrix for the kernel density of event locations (1) in step 2 can be obtained by the plug-in bandwidth selector introduced by Fuentes-Santos et al. (2016). We also need bandwidth matrices, $G_{j}, j=1,2$, for the kernel estimators of the squared density integrals $\psi_{j}, j=1,2$ in (3) to conduct steps 1 and 3.2. Taking into account the closeness between the kernel estimators of the density of event locations of spatial point processes and the density of bivariate random variables, these bandwidths can be obtained by the plug-in algorithm proposed by Chacón and Duong (2010) for integrated density derivatives considering, in this case, order $r=0$.

\section{Simulation study}

We have conducted a simulation study to analyze the performance of the nonparametric test introduced above. We have checked the normality of $\hat{T}$ under the null hypothesis, and compared the probabilities of rejecting $\mathcal{H}_{0}$ provided by the asymptotic and bootstrap calibrations. We have also analyzed the power of the test for different ways and degrees of departure from the null hypothesis.

We first generated inhomogeneous spatial Poisson point processes (IPP1) with first-order intensity functions $\lambda_{j}(x)=a_{j} \exp \left(-3 x_{2}\right), j=1,2$, for any $x=\left(x_{1}, x_{2}\right) \in \mathbb{R}^{2}$ on the unit square. Different values of $a_{j}, j=1,2$ were considered to obtain different proportions of type 1 and type 2 events in order to check whether the asymmetry in the size of the point patterns affects the performance of the test. The alternative hypothesis was generated adding a 
random number of events uniformly distributed on a subregion of the type 2 point pattern as follows

$$
\left\{\begin{array}{l}
\lambda_{1}(x)=a_{1} \exp \left(-3 x_{2}\right) \\
\lambda_{2}(x)=a_{2}\left(\exp \left(-3 x_{2}\right)+\frac{100}{a_{2}} \epsilon\left(\frac{1}{4}\right)^{-2} I_{\left(x_{1}, x_{2}\right) \in[0.5,0.75]^{2}}\right)
\end{array}\right.
$$

Using $a_{1}=a_{2}=1700$ we generate realizations of a bivariate point process with $m_{1}=m_{2}=500 .(1 / 4)^{2}$ in expression (7) is the area of the subregion of the unit square where $100 \epsilon$ events are added to generate realizations of the alternative hypothesis in the balanced design, and $\epsilon$, which takes values between 0.2 and 0.8 at 0.2 intervals, determines the number of events added to the type 2 point process, that is the level of departure from the null hypothesis (Figure 1). These processes are an inhomogeneous and discretized version of the point processes used in Díaz-Avalos et al. (2013) to check the performance of nonparametric separability tests.

We also tested the performance of the test for pairwise comparisons on a multitype inhomogeneous spatial Poisson point process (IPP2) with marginal intensities

$$
\lambda_{j}(x)=a_{j} \phi\left(\epsilon-0.2 x_{2}, 0.02\right), \epsilon_{j}=0.1(j-1) / 8, j=1, \ldots, 9
$$

where $\phi(\mu, \sigma)$ denotes the density function of the univariate normal distribution with mean $\mu$ and variance $\sigma^{2}$, and $a_{j}$ ranked from 500 to 50 at 50 intervals to generate marginal patterns with different sizes. As we can see in Figure 2, where the marginal patterns are labeled from $\mathrm{A}\left(\epsilon_{1}=0\right)$ to I $\left(\epsilon_{9}=0.1\right)$, the high-intensity area moves upwards as $\epsilon$ increases.

We generated 1000 realizations under each one of the different scenarios defined by the values of $\epsilon$ in expressions (7) and (8), and conducted the asymptotic and bootstrap tests based on $\hat{T}$. The bootstrap distribution of $\hat{T}$ under $\mathcal{H}_{0}$ was obtained from $B=1000$ realizations of the null hypothesis $(\epsilon=0)$. The probabilities of rejecting $\mathcal{H}_{0}$ at any significance level, $\alpha$, were computed as the proportion of times the p-value provided by each calibration was smaller than $\alpha$. The simulation study was conducted with the help of the spatstat (Baddeley and Turner, 2005) and $k s$ (Duong, 2013a) packages of R (R Core Team, 2014). 

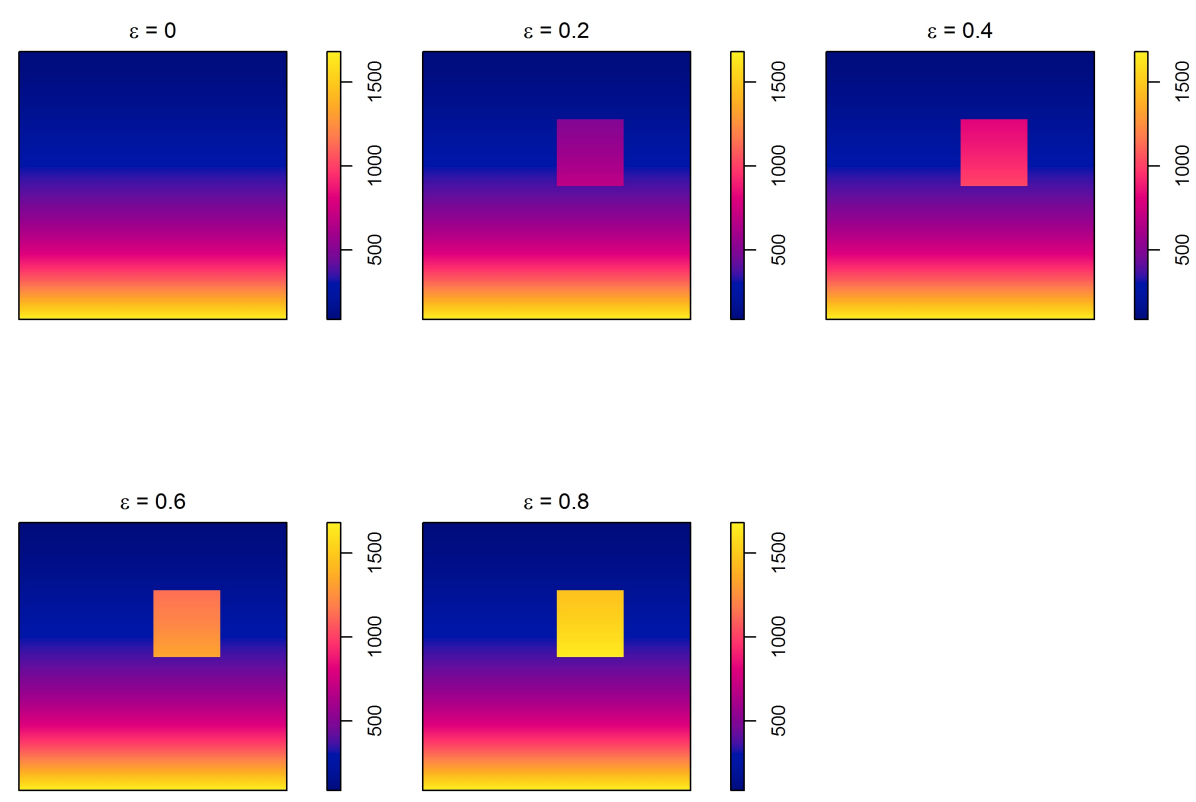

Figure 1: First-order intensities for the type 1 and type 2 events in the bivariate point processes with marginal intensity functions (7), and balanced design. 

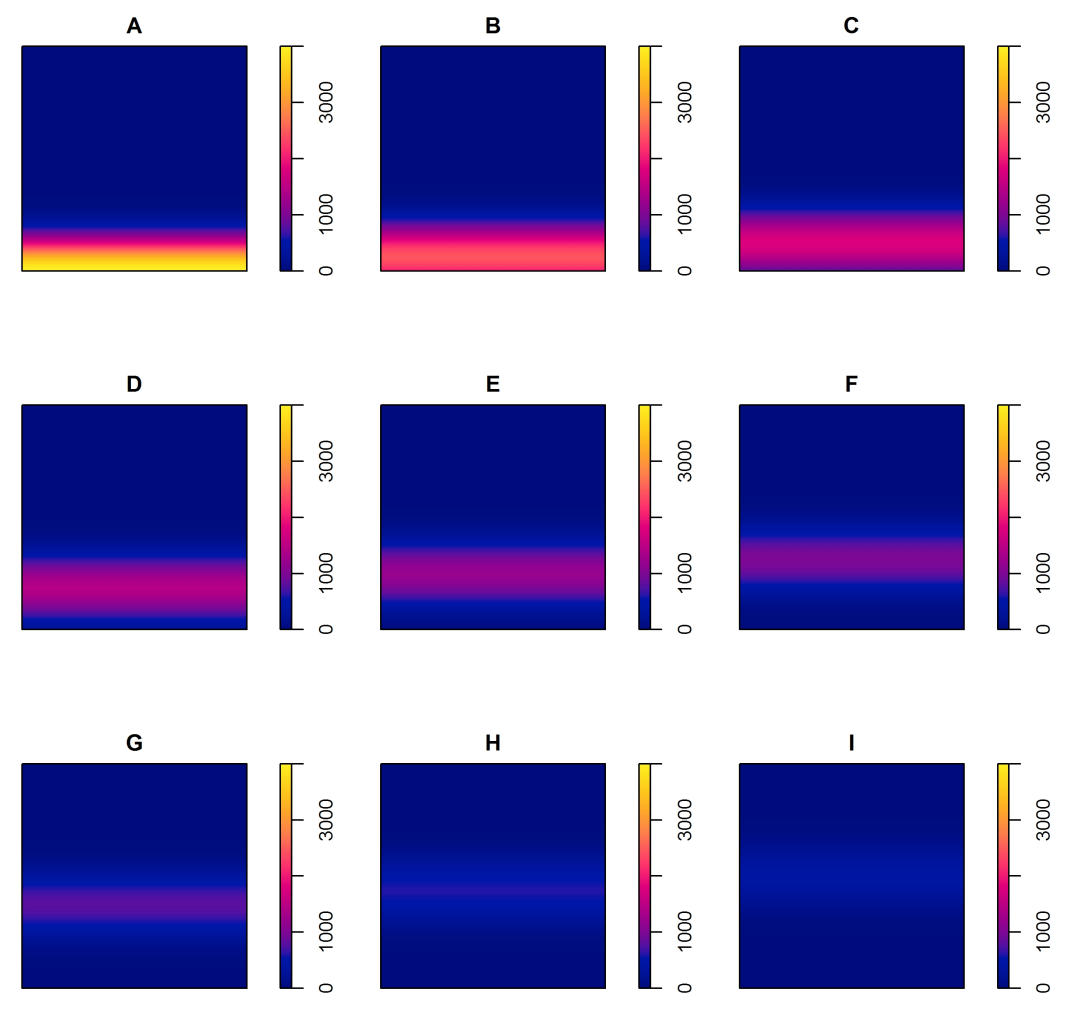

Figure 2: Multitype point process: marginal intensities of the type A-I point processes with first-order intensity functions (8). 
For the spatial point processes with univariate intensities defined in expression (7), Figure 3 and Table 1 show that $\hat{T}$ is far from being normally distributed. In addition the asymptotic calibration overestimates the variance of $\hat{T}$ and leads to a conservative test. The bootstrap calibration provides type I errors close to the nominal significance level, although the test can be slightly conservative for unbalanced designs. As expected, given their performance under $\mathcal{H}_{0}$, the bootstrap test is more powerful than the asymptotic test. The power of the test increases with departure from the null hypothesis, and decreases for unbalanced designs.
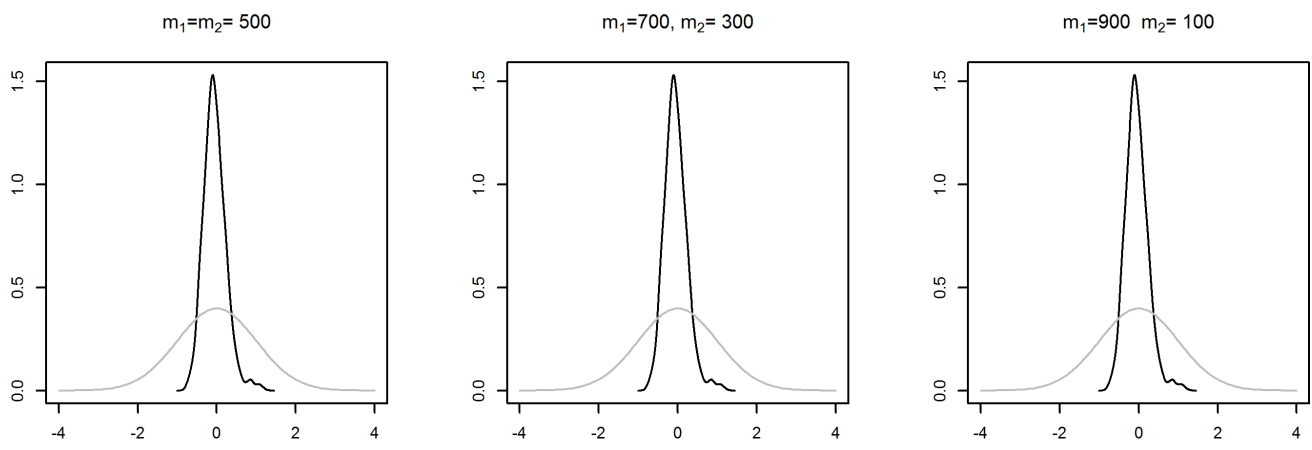

Figure 3: Comparison between the empirical distribution of the standardized test statistic, $\hat{Z}$, for IPP1 (black) and the standard normal distribution (grey).

Figure 4 and Table 2 show that the null distributions of $\hat{T}$ for the pairwise comparisons in IPP2 (Figure 2) are also far from being normal. Table 2 also shows that the bias of $\hat{\mu}_{Z}$ increases as the number of events in each pattern decreases. In contrast, $\hat{\sigma}_{Z}$ approximates 1 as the pattern size decreases, leading to more conservative tests for large patterns. The bootstrap calibration is less affected by the sample sizes, and provides type 1 errors reasonably close to the nominal significance levels. Although, for type $\mathrm{H}$ events the asymptotic calibration performs better than the bootstrap calibration for $\alpha=0.01$, the general behavior of the latter is better under the null hypothesis.

Tables 3 and 4 show that the bootstrap test is more powerful than the asymptotic test. For both calibrations, the power increases with the size 


\begin{tabular}{cccccccccc}
\hline & & \multicolumn{1}{c}{$\alpha=0.05$} & \multicolumn{2}{c}{$\alpha=0.01$} & \multicolumn{3}{c}{$\hat{Z}$} \\
\cline { 5 - 10 } & $\epsilon$ & $N_{2, \epsilon}$ & $\mathrm{A}$ & $\mathrm{B}$ & $\mathrm{A}$ & $\mathrm{B}$ & $\hat{\mu}_{Z}$ & $\hat{\sigma}_{Z}$ & shapiro-test \\
\hline$m_{1}=500$ & 0 & & $<0.001$ & 0.048 & $<0.001$ & 0.007 & -0.05 & 0.29 & $<1 \mathrm{e}-9$ \\
$m_{2}=500$ & 0.2 & 20 & $<0.001$ & 0.103 & $<0.001$ & 0.022 & & & \\
& 0.4 & 40 & 0.015 & 0.315 & 0.002 & 0.134 & & & \\
& 0.6 & 60 & 0.263 & 0.778 & 0.067 & 0.587 & & & \\
& 0.8 & 80 & 0.780 & 0.994 & 0.465 & 0.978 & & & \\
$m_{1}=700$ & 0 & & $<0.001$ & 0.044 & $<0.001$ & 0.009 & -0.04 & 0.30 & $<1 \mathrm{e}-9$ \\
$m_{2}=300$ & 0.2 & 12 & 0.001 & 0.064 & 0.000 & 0.010 & & & \\
& 0.4 & 24 & 0.017 & 0.263 & 0.002 & 0.124 & & & \\
& 0.6 & 36 & 0.137 & 0.718 & 0.032 & 0.517 & & & \\
& 0.8 & 48 & 0.471 & 0.888 & 0.187 & 0.705 & & & \\
$m_{1}=900$ & 0 & & $<0.001$ & 0.035 & $<0.001$ & 0.004 & -0.07 & 0.35 & $<1 \mathrm{e}-9$ \\
$m_{2}=100$ & 0.2 & 4 & 0.004 & 0.081 & $<0.001$ & 0.025 & & & \\
& 0.4 & 8 & 0.011 & 0.080 & 0.001 & 0.022 & & & \\
& 0.6 & 12 & 0.050 & 0.242 & 0.013 & 0.058 & & & \\
& 0.8 & 16 & 0.129 & 0.466 & 0.037 & 0.177 & & & \\
\hline
\end{tabular}

Table 1: Performance of the asymptotic (A) and bootstrap (B) tests based on $\hat{T}$ for IPP1. Probabilities of rejection with the asymptotic and bootstrap distributions of $\hat{T}$ at nominal significance levels $\alpha=0.05$ and $\alpha=0.01$. Empirical mean $\hat{\mu}_{Z}$, and standard deviations $\hat{\sigma}_{Z}$, and p-value of the Shapiro-Wilk test for the standardized test statistic, $\hat{Z}$, under $\mathcal{H}_{0}$. For the alternative hypothesis $\epsilon$ determines the degree of departure from $\mathcal{H}_{0}$ and $N_{2, \epsilon}$ the expected number of events added to the original pattern. 
of the observed patterns. As for IPP1, the probability of rejecting the null hypothesis increases with the discrepancy between the univariate first-order intensities, in fact we only obtain probabilities of rejection lower than 1 for consecutive patterns.

\begin{tabular}{ccccccccc}
\hline & & \multicolumn{2}{c}{$\alpha=0.05$} & \multicolumn{2}{c}{$\alpha=0.01$} & \multicolumn{2}{c}{$\hat{Z}$} \\
\cline { 3 - 10 } & $m_{j}$ & $\mathrm{~A}$ & $\mathrm{~B}$ & $\mathrm{~A}$ & $\mathrm{~B}$ & $\hat{\mu}_{Z}$ & $\hat{\sigma}_{Z}$ & shapiro-test \\
\hline $\mathrm{A}$ & 500 & 0.001 & 0.067 & $<0.001$ & 0.016 & -0.06 & 0.42 & $<1 \mathrm{e}-9$ \\
$\mathrm{~B}$ & 450 & 0.005 & 0.044 & 0.003 & 0.013 & -0.09 & 0.56 & $<1 \mathrm{e}-9$ \\
$\mathrm{C}$ & 400 & 0.012 & 0.037 & 0.002 & 0.004 & -0.16 & 0.77 & $<1 \mathrm{e}-9$ \\
$\mathrm{D}$ & 350 & 0.020 & 0.052 & 0.008 & 0.009 & -0.15 & 0.88 & $<1 \mathrm{e}-9$ \\
$\mathrm{E}$ & 300 & 0.018 & 0.059 & 0.007 & 0.014 & -0.23 & 0.92 & $<1 \mathrm{e}-9$ \\
$\mathrm{~F}$ & 250 & 0.022 & 0.045 & 0.007 & 0.014 & -0.24 & 1.00 & $<1 \mathrm{e}-9$ \\
$\mathrm{G}$ & 200 & 0.021 & 0.088 & 0.005 & 0.016 & -0.28 & 0.92 & $<1 \mathrm{e}-9$ \\
$\mathrm{H}$ & 150 & 0.025 & 0.045 & 0.009 & 0.006 & -0.27 & 0.96 & $<1 \mathrm{e}-9$ \\
$\mathrm{I}$ & 100 & 0.023 & 0.062 & 0.010 & 0.014 & -0.24 & 0.96 & $<1 \mathrm{e}-9$ \\
\hline
\end{tabular}

Table 2: Performance of the test under the null hypothesis for multitype inhomogeneous Poisson point processes (IPP2). Probabilities of rejection with the asymptotic and bootstrap distributions of $\hat{T}$ at nominal significance levels $\alpha=0.05$ and $\alpha=0.01$. Empirical mean $\hat{\mu}_{Z}$, and standard deviations $\hat{\sigma}_{Z}$ for the standardized test statistic, and p-value of the Shapiro-Wilk test.

This simulation study shows the good performance of the nonparametric test introduced in this paper. Comparison between calibration of its null distribution supports the need of a bootstrap resampling, though the asymptotic test performed reasonably well for IPP2, perhaps because normal distributions were involved in the first-order intensities of the simulated patterns. Finally the power of the test increases with the departure from the null hypothesis, as expected, and decreases for small patterns and unbalanced designs.

\section{Comparison between spatial patterns of wildfires in Galicia}

Wildfire is the most ubiquitous natural disturbance in the world and represents a problem of considerable social and environmental importance. Particularly in Galicia (NW Spain) arson wildfires are the main cause of 

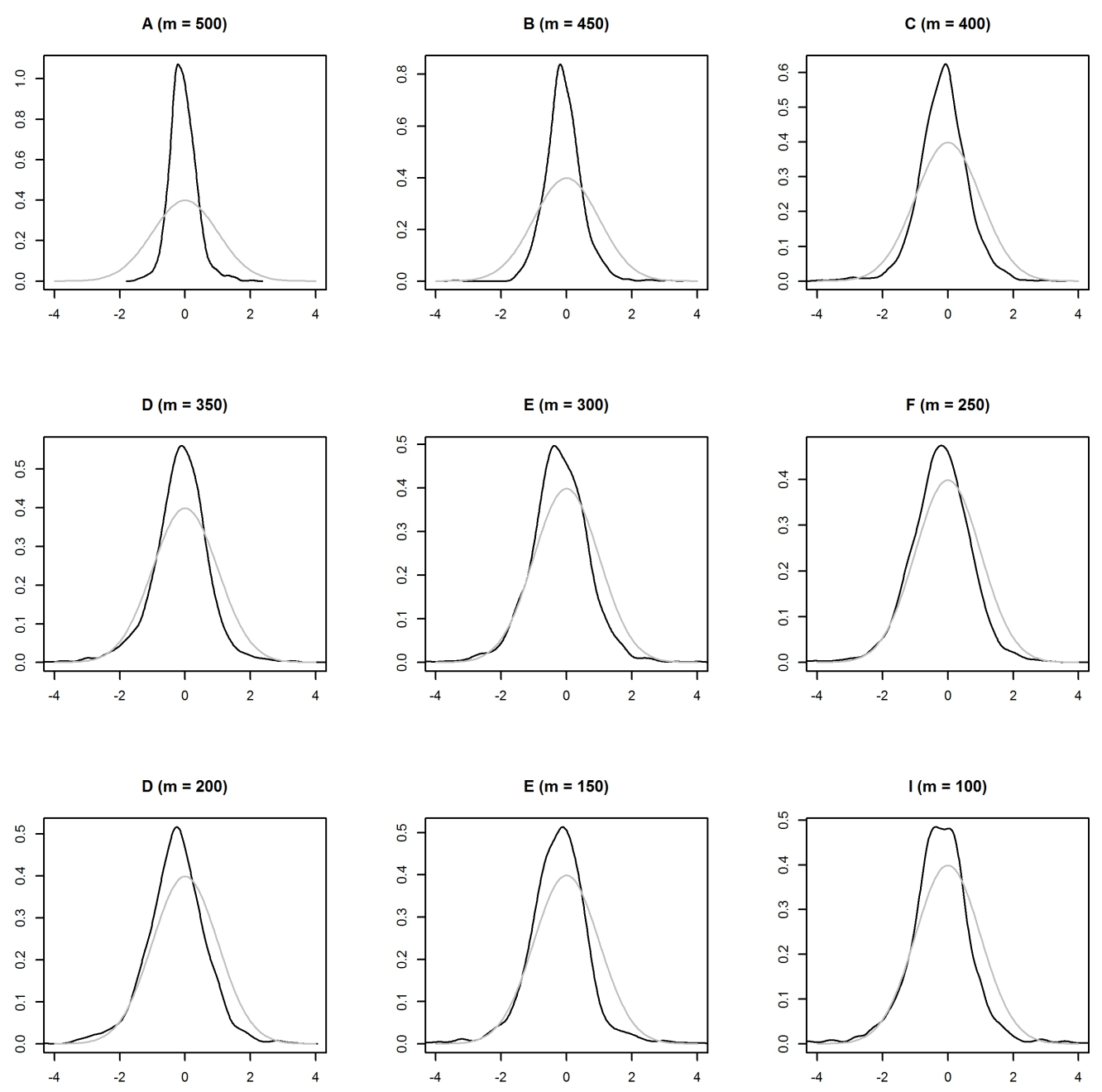

Figure 4: Comparison between the empirical distribution of the standardized test statistic, $Z$, for IPP2 (black) and the standard normal distribution (grey). 


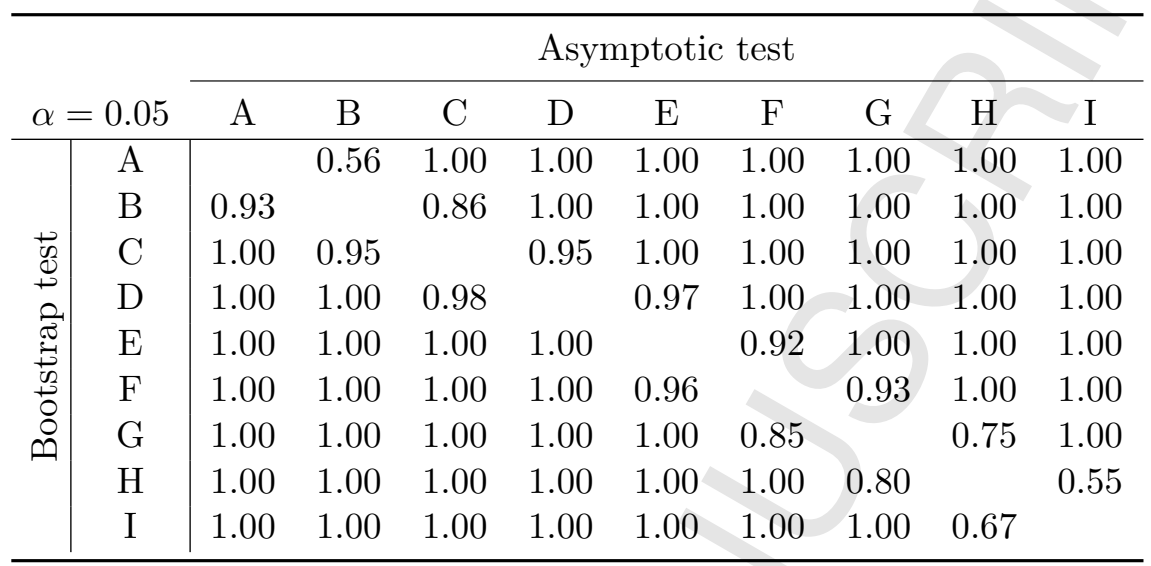

Table 3: Power of the $T$-test at significance level $\alpha=0.05$, for 1000 realizations of multitype point processes (IPP2). Comparison between asymptotic (upper triangular) and bootstrap (lower triangular) tests.

\begin{tabular}{|c|c|c|c|c|c|c|c|c|c|c|}
\hline \multirow{2}{*}{\multicolumn{2}{|c|}{$\alpha=0.01$}} & \multicolumn{9}{|c|}{ Asymptotic test } \\
\hline & & $\mathrm{A}$ & $\mathrm{B}$ & $\mathrm{C}$ & $\mathrm{D}$ & $\mathrm{E}$ & $\mathrm{F}$ & $\mathrm{G}$ & $\mathrm{H}$ & $\mathrm{I}$ \\
\hline \multirow{9}{*}{$\begin{array}{l}w \\
\mathbb{D} \\
+ \\
0 \\
0 \\
0 \\
0 \\
0 \\
0 \\
0 \\
0 \\
0\end{array}$} & $\mathrm{~A}$ & & 0.27 & 1.00 & 1.00 & 1.00 & 1.00 & 1.00 & 1.00 & 1.00 \\
\hline & $\mathrm{B}$ & 0.81 & & 0.65 & 1.00 & 1.00 & 1.00 & 1.00 & 1.00 & 1.00 \\
\hline & $\mathrm{C}$ & 1.00 & 0.82 & & 0.87 & 1.00 & 1.00 & 1.00 & 1.00 & 1.00 \\
\hline & $\mathrm{D}$ & 1.00 & 1.00 & 0.92 & & 0.88 & 1.00 & 1.00 & 1.00 & 1.00 \\
\hline & $\mathrm{E}$ & 1.00 & 1.00 & 1.00 & 0.96 & & 0.81 & 1.00 & 1.00 & 1.00 \\
\hline & $\mathrm{F}$ & 1.00 & 1.00 & 1.00 & 1.00 & 0.87 & & 0.70 & 1.00 & 1.00 \\
\hline & $\mathrm{G}$ & 1.00 & 1.00 & 1.00 & 1.00 & 1.00 & 0.82 & & 0.55 & 1.00 \\
\hline & $\mathrm{H}$ & 1.00 & 1.00 & 1.00 & 1.00 & 1.00 & 1.00 & 0.64 & & 0.35 \\
\hline & $\mathrm{I}$ & 1.00 & 1.00 & 1.00 & 1.00 & 1.00 & 1.00 & 1.00 & 0.52 & \\
\hline
\end{tabular}

Table 4: Power of the $T$-test at significance level $\alpha=0.01$, for 1000 realizations of multitype point processes (IPP2). Comparison between asymptotic (upper triangular) and bootstrap (lower triangular) tests. 
forest destruction. This problem has motivated an increasing interest in the development of statistical techniques to understand and analyze the behavior of wildfires in this region (Boubeta et al., 2015; Fuentes-Santos et al., 2013; Ríos-Pena et al., 2015).

In this section we consider the dataset comprising the spatial locations and time of occurrence for the wildfires registered in Galicia during 2006. In agreement with the classification of the Galician government (PLADIGA, Consellería do Medio Rural (2016)), wildfires were grouped into three types according to the burned area $(S): 4800$ small fires $(S<1$ ha), 1767 regular fires $(1 \leq S<25 \mathrm{ha})$, and 337 large wildfires $(S \geq 25 \mathrm{ha})$, thus the majority of wildfires in this region, above $70 \%$ in 2006, had less than 1 ha affected, while large fires represented less than $5 \%$ of wildfires. We also classified wildfires into five groups according with their cause: 5090 arson, 120 natural, 400 due to negligence, 475 reproductions, and 819 with unknown cause. We observe a high incidence of arson wildfires, over $73 \%$, in contrast with the low risk of fires with natural cause, below $2 \%$.

Both the high incidence of arson and small wildfires are particular features of Galicia, in contrast with other regions of Spain (Juan et al., 2012; Serra et al., 2014). We have applied the nonparametric procedure introduced in this work to test whether, in addition to the clear differences in fire incidence, wildfires with different burned area or cause also have a different spatial distribution.

Pairwise comparisons between the first-order intensity functions of small, regular and large wildfires (Figure 5) confirmed that the three point patterns have different spatial distribution ( $\mathrm{p}$-values $<0.001$ with both asymptotic and bootstrap calibrations). Pairwise comparison between the five causes of wildfires (Figure 6) also detected significant differences between their firstorder intensities ( $\mathrm{p}$-value $<0.001$ with both asymptotic and bootstrap calibrations).

The test statistic $\hat{T}$ can also be used to test the separability assumption (Díaz-Avalos et al., 2013; Schoenberg, 2004) on spatio-temporal point processes with discrete temporal component. For instance, we can stratify the spatio-temporal pattern of wildfires registered in 2006 by months and test whether their spatial structure changes over time. The number of wildfires 

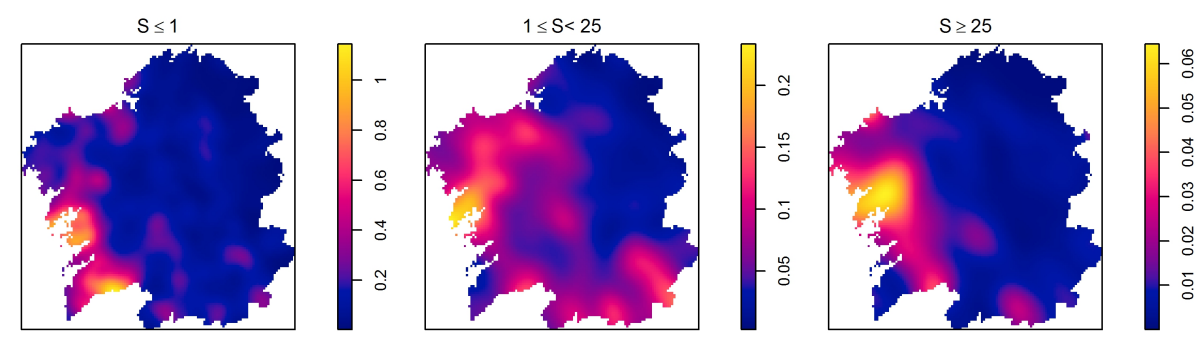

Figure 5: Kernel intensity estimator of the wildfires registered in Galicia during 2006 by burned area (different scales).
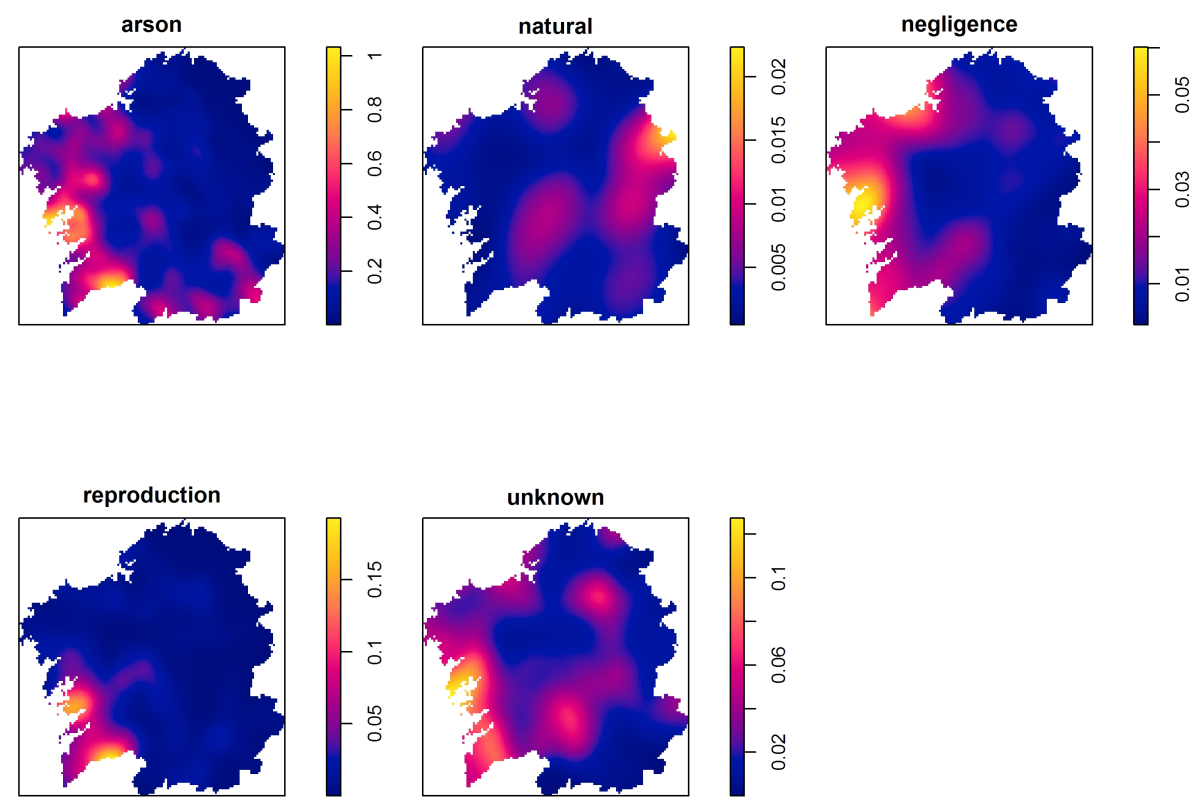

Figure 6: Kernel intensity estimator of the wildfires registered in Galicia during 2006 by cause (different scales). 
shows an important seasonal variability, with maximum values in summer (2230 ignition points in August) and minimum values in autumn (6 ignition points in October). We have considered the wildfires registered between January and September, as the small number of events registered during the last 3 months do not allow estimating the corresponding first-order intensity functions.

The kernel intensity estimators in Figure 7 show that the region with high incidence of wildfires shifted from the southern area of Galicia, from January to March, to the west coast, which registered the highest fire intensity during summer. The asymptotic and bootstrap tests (Table 5) confirmed the temporal variability in the spatial patterns of ignition points, being April and May the only pair of months with homogeneous spatial structure.

\begin{tabular}{|c|c|c|c|c|c|c|c|c|c|c|c|}
\hline \multirow{2}{*}{\multicolumn{3}{|c|}{$\mathrm{n}$}} & \multicolumn{9}{|c|}{ Asymptotic test } \\
\hline & & & $\mathrm{J}$ & $\mathrm{F}$ & $\mathrm{Mr}$ & A & My & Jn & $\mathrm{Jl}$ & $\mathrm{Ag}$ & $\mathrm{S}$ \\
\hline & & & 60 & 659 & 89 & 260 & 441 & 638 & 1789 & 2238 & 669 \\
\hline & $\mathrm{J}$ & 60 & & 0 & 0 & 0.025 & 0 & 0.007 & 0.001 & 0 & 0 \\
\hline & $\mathrm{F}$ & 659 & 0 & & 0 & 0 & 0 & 0 & 0 & 0 & 0 \\
\hline$\vec{y}$ & $\mathrm{Mr}$ & 89 & 0 & 0.014 & & 0 & 0 & 0 & 0 & 0 & 0 \\
\hline & A & 268 & 0 & 0 & 0 & & 0.215 & 0 & 0 & 0 & 0 \\
\hline $\mathbb{Z}^{\mathbb{Z}}$ & My & 441 & 0 & 0 & 0 & 0.09 & & 0 & 0 & 0 & 0 \\
\hline$\frac{\pi}{0}$ & $\mathrm{Jn}$ & 638 & 0 & 0 & 0 & 0 & 0 & & 0 & 0 & 0 \\
\hline$\stackrel{0}{0}$ & $\mathrm{Jl}$ & 1789 & 0 & 0 & 0 & 0 & 0 & 0 & & 0 & 0 \\
\hline & $\mathrm{Ag}$ & 2238 & 0 & 0 & 0 & 0 & 0 & 0 & 0 & & 0 \\
\hline & $\mathrm{S}$ & 669 & 0 & 0 & 0 & 0 & 0 & 0 & 0 & 0 & \\
\hline
\end{tabular}

Table 5: P-values of the pairwise comparison between months of the spatial distribution of wildfires registered in Galicia during 2006. Asymptotic calibration in the upper triangular and bootstrap calibration in the lower triangular $(0$ indicate p-value $<0.001)$. We considered the period January-September as less than 30 fires by month were observed from October onwards. $n$ is the number of wildfires registered each month.

\section{Conclusions}

Up to date the similarity between two spatial point processes has been addressed through distance-based (Hahn, 2012) or area-based (Alba-Fernández 

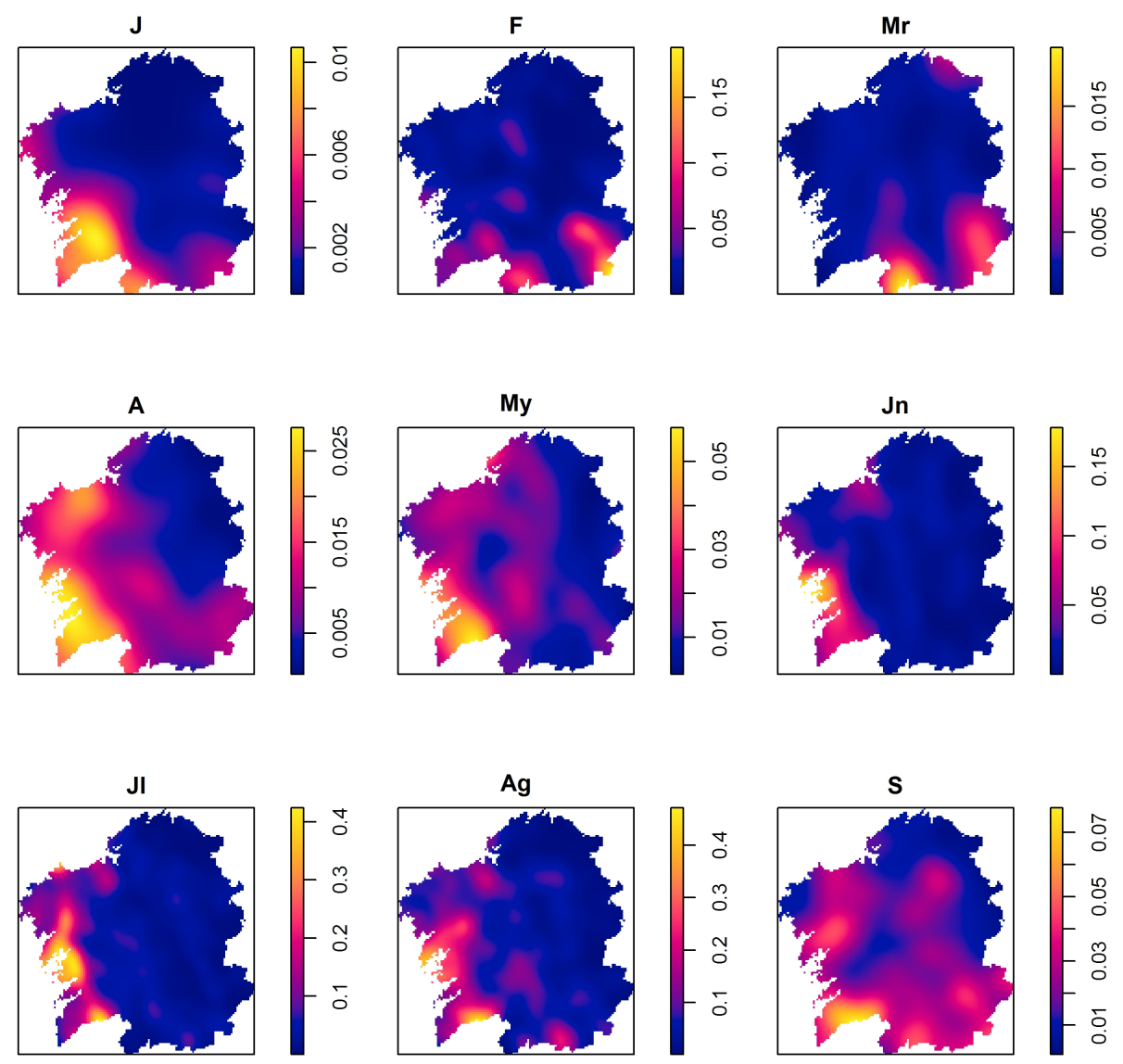

Figure 7: First-order intensity functions for the spatial patterns of wildfire registered in Galicia from January to September 2006 (different scales). 
et al., 2016; Andresen, 2009) tests. However, comparison of their first-order properties seems to be the natural way to deal with this issue. For this reason, following the idea of Duong et al. (2012) for comparison of multivariate random distributions, we have proposed a nonparametric test based on a square discrepancy between densities of event locations to compare the firstorder structure of inhomogeneous spatial point patterns. Assuming that the point processes are Poisson and considering regularity conditions analogous to those assumed by Duong et al. (2012) in the multivariate framework, we have seen that the statistical test is asymptotically normal. In addition, taking into account the limitations of asymptotic calibrations for small sample sizes in other areas of statistics we have proposed an alternative calibration procedure through smooth bootstrap.

The simulation study has shown that the asymptotic test can be quite conservative, while the bootstrap procedure provides reasonable estimates of the nominal significance level and is more powerful than the asymptotic test. These results point out the need of using the computational demanding bootstrap calibration. The test was able to detect different ways of departure from the null hypothesis, and its power increases with departure from $\mathcal{H}_{0}$. However, the power of the test reduces for small point patterns and for unbalanced designs.

In the application to the analysis of wildfires the asymptotic and bootstrap tests lead to the same conclusions. However, in view of the simulation study we recommend using the bootstrap calibration. The results of the pairwise comparisons among sizes and causes of wildfires indicate that different covariates and models should be used to predict the occurrence of each type of wildfire. For instance, the different spatial distribution of natural and arson fires, along with the low incidence of fires with natural cause, warns against using wildfires risk indexes based on meteorological conditions in Galicia, although their good performance has been tested in other countries. We have also seen that the test introduced in this work can be used to test the separability assumption, i.e. to test whether the spatial distribution of events varies over time, which is one of the main issues when dealing with spatio-temporal point processes (Díaz-Avalos et al., 2013; Schoenberg, 2004). For this purpose we should stratify the temporal component into subgroups to generate a multitype point process. Arranging the temporal component into subgroups is recommended when data are collected at coarse level or to 
avoid the negative effect of temporal sparseness.

Along this work we have introduced a nonparametric test to check whether two spatial point patterns have the same spatial distribution. The simulation study and application to real data confirm the good performance of the test. Future research can address two possible extensions of this work. On one hand, we can be interested on testing whether $p>2$ spatial point processes have the same spatial structure. For this purpose we need to extend the pairwise discrepancy measure and the bootstrap calibration procedure introduced in this work to the multitype framework. On the other hand, we have developed a test that analyzes the global discrepancy between the first-order structure of two spatial point processes. We may also be interested on measuring local discrepancies between first-order intensity functions, for instance we can wonder whether the risk of arson wildfires at a given location is higher than the risk of natural wildfires. Considering once again the equivalence between the bivariate density function and the density of event locations, we can extend the nonparametric test introduced by Duong (2013b) to measure local discrepancies between multivariate density functions to the spatial point process framework. 


\section{Appendix A. Proof of Theorem 1}

\section{Expected value}

From expression (3) we have that

$$
\mathbb{E} \hat{T}=\mathbb{E} \hat{\psi}_{1}+\mathbb{E} \hat{\psi}_{2}-\mathbb{E} \hat{\psi}_{12}-\mathbb{E} \hat{\psi}_{21}
$$

For $j=1,2$ we have

$$
\begin{aligned}
\hat{\psi}_{j} & =N_{j}^{-2} \sum_{i_{1}=1}^{N_{j}} \sum_{i_{2}=1}^{N_{j}} k_{G_{1}}\left(\mathbf{x}_{\mathbf{i}_{1}}-\mathbf{x}_{\mathbf{i}_{\mathbf{2}}}\right) I\left(N_{j}>0\right) \\
& =N_{j}^{-1} k_{G_{j}}(0) I\left(N_{j}>0\right)+N_{j}^{-2} \sum_{i_{1}=1}^{N_{j}} \sum_{i_{1} \neq i_{2}} k_{G_{j}}\left(\mathbf{x}_{\mathbf{i}_{\mathbf{1}}}-\mathbf{x}_{\mathbf{i}_{\mathbf{2}}}\right) I\left(N_{j}>0\right)
\end{aligned}
$$

Given that $\mathbf{X}_{j}$ is Poisson and $k$ is a measurable function we can use the same properties as those used by Cucala (2006) and Fuentes-Santos et al. (2016) to develop the asymptotic performance of the kernel estimator of the density of event locations to obtain

$$
\mathbb{E} \hat{\psi}_{j}=A\left(m_{j}\right) k_{G_{j}}(0)+\mathbb{E}\left[\mathbb{E}\left[\left(1-N_{j}^{-1}\right) k_{G_{j}}\left(\mathbf{x}_{\mathbf{1}}-\mathbf{x}_{\mathbf{2}}\right) \mid N_{j}>0\right]\right]
$$

where $A\left(m_{j}\right)=\mathbb{E}\left[\frac{1}{N_{j}} I\left(N_{j}>0\right)\right]=e^{-m_{j}} \sum_{k=1}^{\infty} \frac{m^{k}}{k k !}<e^{-m_{j}} \sum_{k=0}^{\infty} \frac{2 m_{j}^{k}}{(k+1) !}=$ $2 / m_{j} \rightarrow 0$ when $m_{j} \rightarrow \infty$. The second term in the right hand side of expression (A.2) is

$$
\begin{aligned}
& \mathbb{E}\left[\mathbb{E}\left[\left(1-N_{j}^{-1}\right) k_{G_{j}}\left(\mathbf{x}_{\mathbf{1}}-\mathbf{x}_{\mathbf{2}}\right) \mid N_{j}>0\right]\right] \\
& =\sum_{l=1}^{\infty}\left(1-\frac{1}{l}\right) \int k_{G}(x-y) \lambda_{0 j}(x) \lambda_{0 j}(y) d x d y P\left(N_{j}=l\right) \\
& =\left(1-e^{-m_{j}}-A\left(m_{j}\right)\right)\left[\psi_{j}+\frac{\mu_{2}(k)}{2} \operatorname{vec}^{T} G_{j} \psi_{j}^{(2)}+o\left(\operatorname{tr} G_{j}\right)\right]
\end{aligned}
$$

where $v e c A$ is the vector operator applied to the symmetric matrix $A$, that is the column vector containing all entries in $A, \mu_{2}(k)<\infty$ is a positive number such that $\int_{\mathbb{R}^{2}} u u^{T} k(u) d u=\mu_{2}(k) I_{2}$, where $I_{2}$ is the two-dimensional identity matrix, and $\psi_{j}^{(2)}=\int D^{2} \lambda_{0 j}(x) \lambda_{0 j}(x) d x$, being $D^{2} \lambda_{0 j}(\cdot)$ the Hessian 
matrix of $\lambda_{0 j}(\cdot)$.

Plugging (A.3) into expression (A.2) we obtain

$$
\mathbb{E} \hat{\psi}_{j}=A\left(m_{j}\right) k_{G_{j}}(0)+\left(1-A\left(m_{j}\right)\right)\left[\psi_{j}+\frac{\mu_{2}(k)}{2} \operatorname{vec}^{T} G_{j} \psi_{j}^{(2)}\right](1+o(1))
$$

which when $m_{j} \rightarrow \infty$ yields

$$
\mathbb{E} \hat{\psi}_{j}=\psi_{j}+\frac{\mu_{2}(k)}{2} \operatorname{vec}^{T} G_{j} \psi_{j}^{(2)}(1+o(1))
$$

For the cross-product integrated densities of event locations we have

$$
\begin{aligned}
\mathbb{E} \hat{\psi}_{l_{1} l_{2}} & =\mathbb{E}\left[\mathbb{E}\left[k_{G_{l_{1}}}\left(\mathbf{x}_{\mathbf{l}_{\mathbf{1}}, \mathbf{1}}-\mathbf{x}_{\mathbf{l}_{\mathbf{2}}, \mathbf{1}}\right) \mid N_{l_{1}}>0, N_{l_{2}}>0\right]\right] \\
& =\mathbb{E}\left[\int k_{G_{l_{1}}}\left(\mathbf{x}_{\mathbf{l}_{1}, \mathbf{1}}-y\right) \lambda_{0 l_{2}}(y) d y d x \mid N_{l_{2}}>0, N_{l_{1}}>0\right] \\
& =\left(1-e^{-m_{l_{1}}}\right)\left(1-e^{-m_{l_{2}}}\right) \int k_{G_{l_{1}}}(x-y) \lambda_{0 l_{2}}(y) \lambda_{0 l_{1}}(x) d y d x
\end{aligned}
$$

Applying a second-order Taylor expansion we obtain

$$
\mathbb{E} \hat{\psi}_{l_{1} l_{2}}=\left(1-e^{-m_{l_{1}}}\right)\left(1-e^{-m_{l_{2}}}\right)\left[\psi_{l_{1} l_{2}}+\frac{\mu_{2}(k)}{2} \operatorname{vec}^{T} G_{l_{1}} \int D^{2} \lambda_{0 l_{1}}(x) \lambda_{0 l_{2}}(x) d x+o\left(\operatorname{tr} G_{l_{1}}\right)\right]
$$

Thus

$$
\mathbb{E} \hat{\psi}_{l_{1} l_{2}}=\left(\psi_{l_{1} l_{2}}+\frac{\mu_{2}(k)}{2} \operatorname{vec}^{T} G_{j_{1}} \int D^{2} \lambda_{0 l_{1}}(x) \lambda_{0 l_{2}}(x) d x\right)(1+o(1))
$$

Therefore if we plug expressions (A.4) and (A.5) into (A.1) we obtain

$$
\begin{aligned}
\mathbb{E} \hat{T} & =\mathbb{E} \hat{\psi}_{1}+\mathbb{E} \hat{\psi}_{2}-\mathbb{E} \hat{\psi}_{12}-\mathbb{E} \hat{\psi}_{21}=\psi_{1}+\psi_{2}-\left(\psi_{12}+\psi_{21}\right) \\
& +\left(A\left(m_{1}\right)\left|G_{1}\right|^{-1 / 2}+A\left(m_{2}\right)\left|G_{2}\right|^{-1 / 2}\right) k(0) \\
& +\frac{\mu_{2}(k)}{2}\left[v e c^{T} G_{1} \psi_{1}^{(2)}+\operatorname{vec}^{T} G_{2} \psi_{2}^{(2)}-v e c^{T} G_{1} \psi_{12}^{(2)}-v e c^{T} G_{2} \psi_{21}^{(2)}\right](1+o(1))
\end{aligned}
$$

Under the null hypothesis $\mathcal{H}_{0}: \lambda_{01}=\lambda_{02}=\lambda_{0}$ and, consequently $\psi_{1}=$ $\psi_{2}=\psi_{12}=\psi_{21}=\psi$. Thus, the terms with order $O\left(\operatorname{tr} G_{1}\right)$ and $O\left(\operatorname{tr} G_{2}\right)$ in expression (A.6) vanish, and the mean of $\hat{T}$ reduces to expression (5)

$$
\mathbb{E} \hat{T}=\mu_{\hat{T}}=\left(A\left(m_{1}\right)\left|G_{1}\right|^{-1 / 2}+A\left(m_{2}\right)\left|G_{2}\right|^{-1 / 2}\right) k(0)
$$




\section{$\underline{\text { Variance }}$}

The variance of $\hat{T}$ is

$$
\begin{aligned}
\operatorname{Var} \hat{T} & =\operatorname{Var}\left(\hat{\psi}_{1}+\hat{\psi}_{2}-\left(\hat{\psi}_{12}+\hat{\psi}_{21}\right)\right) \\
& =\operatorname{Var} \hat{\psi}_{1}+\mathbb{V} a r \hat{\psi}_{2}+\operatorname{Var} \hat{\psi}_{12}+\mathbb{V} \operatorname{ar} \hat{\psi}_{21}-2 \operatorname{Cov}\left(\hat{\psi}_{1}, \hat{\psi}_{12}\right)-2 \operatorname{Cov}\left(\hat{\psi}_{1}, \hat{\psi}_{21}\right) \\
& -2 \operatorname{Cov}\left(\hat{\psi}_{2}, \hat{\psi}_{12}\right)-2 \operatorname{Cov}\left(\hat{\psi}_{2}, \hat{\psi}_{21}\right)+2 \operatorname{Cov}\left(\hat{\psi}_{12}, \hat{\psi}_{21}\right)
\end{aligned}
$$

as $\operatorname{Cov}\left(\hat{\psi}_{1}, \hat{\psi}_{2}\right)=0$ given that $\mathbf{X}_{\mathbf{1}}$ and $\mathbf{X}_{\mathbf{2}}$ are independent point processes.

For $j=1,2$, the law of the total variance yields

$$
\begin{aligned}
\operatorname{Var} \hat{\psi}_{j} & =\operatorname{Var}\left[N_{j}^{-2} \sum_{i_{1}=1}^{N_{j}} \sum_{i_{2} \neq i_{1}} k_{G_{j}}\left(\mathbf{x}_{\mathbf{i}_{\mathbf{1}}}-\mathbf{x}_{\mathbf{i}_{\mathbf{2}}}\right) I\left(N_{j}>0\right)\right] \\
& =\mathbb{E}\left[\mathbb{V} a r\left[N_{j}^{-2} \sum_{i_{1}=1}^{N_{j}} \sum_{i_{2} \neq i_{1}} k_{G_{j}}\left(\mathbf{x}_{\mathbf{i}_{\mathbf{1}}}-\mathbf{x}_{\mathbf{i}_{\mathbf{2}}}\right) \mid N_{j}>0\right]\right] \\
& +\operatorname{Var}\left[\mathbb{E}\left[N_{j}^{-2} \sum_{i_{1}=1}^{N_{j}} \sum_{i_{2} \neq i_{1}} k_{G_{j}}\left(\mathbf{x}_{\mathbf{i}_{1}}-\mathbf{x}_{\mathbf{i}_{\mathbf{2}}}\right) \mid N_{j}>0\right]\right]
\end{aligned}
$$

Using the same arguments as in Wand and Jones (1995), p. 69, the variance in the first summand of expression (A.8) is

$$
\begin{aligned}
& \operatorname{Var}\left[N_{j}^{-2} \sum_{i_{1}=1}^{N_{j}} \sum_{i_{2} \neq i_{1}} k_{G_{j}}\left(\mathbf{x}_{\mathbf{i}_{\mathbf{1}}}-\mathbf{x}_{\mathbf{i}_{\mathbf{2}}}\right) \mid N_{j}>0\right]=2 N_{j}^{-3}\left(N_{j}-1\right) \mathbb{V a r}\left[k_{G_{j}}^{2}\left(\mathbf{x}_{\mathbf{1}}-\mathbf{x}_{\mathbf{2}}\right)\right] \\
& +4 N_{j}^{-3}\left(N_{j}-1\right)\left(N_{j}-2\right) \mathbb{C o v}\left[k_{G_{j}}\left(\mathbf{x}_{\mathbf{1}}-\mathbf{x}_{\mathbf{2}}\right) k_{G_{j}}\left(\mathbf{x}_{\mathbf{2}}-\mathbf{x}_{\mathbf{3}}\right)\right] \\
& \approx 2 N_{j}^{-2}\left(\mathbb{E}\left[k_{G_{j}}^{2}\left(\mathbf{x}_{\mathbf{1}}-\mathbf{x}_{\mathbf{2}}\right)\right]-\mathbb{E}^{2}\left[k_{G_{j}}^{2}\left(\mathbf{x}_{\mathbf{1}}-\mathbf{x}_{\mathbf{2}}\right)\right]\right) \\
& +4 N_{j}^{-1}\left(\mathbb{E}\left[k_{G_{j}}\left(\mathbf{x}_{\mathbf{1}}-\mathbf{x}_{\mathbf{2}}\right) k_{G_{j}}\left(\mathbf{x}_{\mathbf{2}}-\mathbf{x}_{\mathbf{3}}\right)\right]-\mathbb{E}\left[k_{G_{j}}\left(\mathbf{x}_{\mathbf{1}}-\mathbf{x}_{\mathbf{2}}\right)\right] \mathbb{E}\left[k_{G_{j}}\left(\mathbf{x}_{\mathbf{2}}-\mathbf{x}_{\mathbf{3}}\right)\right]\right)
\end{aligned}
$$

using some Taylor expansions we obtain

$$
\begin{aligned}
\mathbb{E}\left[k_{G_{j}}^{2}\left(\mathbf{x}_{\mathbf{1}}-\mathbf{x}_{\mathbf{2}}\right)\right] & =\iint k_{G_{j}}^{2}(x-y) \lambda_{0 j}(x) \lambda_{0 j}(y) d x d y \\
& =\left|G_{j}\right|^{-1 / 2} \int k^{2}(u) d u\left(\int \lambda_{0 j}^{2}(x) d x+O(\operatorname{tr} G)\right) \\
& =\left|G_{j}\right|^{-1 / 2} \psi_{j} R(k)(1+o(1))
\end{aligned}
$$


where $R(k)=\int k^{2}(u) d u$, and

$$
\begin{aligned}
\mathbb{E}\left[k_{G_{j}}\left(\mathbf{x}_{\mathbf{1}}-\mathbf{x}_{\mathbf{2}}\right) k_{G_{j}}\left(\mathbf{x}_{\mathbf{2}}-\mathbf{x}_{\mathbf{3}}\right)\right] & =\iiint k_{G_{j}}(\mathbf{x}-\mathbf{y}) k_{G_{j}}(\mathbf{y}-\mathbf{z}) \lambda_{0 j}(x) \lambda_{0 j}(y) \lambda_{0 j}(z) d x d y d z \\
& =\iiint k(u) k(v) \lambda_{0 j}\left(y+G^{1 / 2} u\right) \lambda_{0 j}(y) \lambda_{0 j}\left(y-G^{1 / 2} v\right) d u d v d y \\
& =\int \lambda_{0 j}^{3}(x) d x(1+o(1))
\end{aligned}
$$

and, as seen above $\mathbb{E}\left[k_{G_{j}}\left(\mathbf{x}_{\mathbf{1}}-\mathbf{x}_{\mathbf{2}}\right)\right]=\int \lambda_{0 j}^{2}(x) d x(1+o(1))$.

Plugging these values into expression (A.9), and using the properties of measurable functions of a Poisson point process (Cucala, 2006), we obtain the following expression for the first summand of (A.8)

$$
\begin{aligned}
& \mathbb{E}\left[\operatorname{Var}\left[N_{j}^{-2} \sum_{i_{1}=1}^{N_{j}} \sum_{i_{2} \neq i_{1}} k_{G_{j}}\left(\mathbf{x}_{\mathbf{i}_{\mathbf{1}}}-\mathbf{x}_{\mathbf{i}_{\mathbf{2}}}\right) \mid N_{j}>0\right]\right] \\
& =2 \mathbb{E}\left[N_{j}^{-2}\left(\left|G_{j}\right|^{-1 / 2} \psi_{j} R(k)-\psi_{j}^{2}\right)(1+o(1)) \mid N_{j}>0\right] \\
& +4 \mathbb{E}\left[N_{j}^{-1}\left(\int \lambda_{0 j}^{3}(x) d x-\psi_{j}^{2}\right)(1+o(1)) \mid N_{j}>0\right] \\
& =2 \sum_{l * 1}^{\infty} \frac{1}{l^{2}} P\left(N_{j}=l\right)\left(\left|G_{j}\right|^{-1 / 2} \psi_{j} R(k)-\psi_{j}^{2}\right)(1+o(1)) \\
& +4 \sum_{l * 1}^{\infty} \frac{1}{l} P\left(N_{j}=l\right)\left(\int \lambda_{0 j}^{3}(x) d x-\psi_{j}^{2}\right)(1+o(1)) \\
& \left.=2 B\left(m_{j}\right)\right)\left(\left|G_{j}\right|^{-1 / 2} \psi_{j} R(k)-\psi_{j}^{2}\right)(1+o(1))+4 A\left(m_{j}\right)\left(\int \lambda_{0 j}^{3}(x) d x-\psi_{j}^{2}\right)(1+o(1))
\end{aligned}
$$

where $B\left(m_{j}\right)=\mathbb{E}\left[\frac{1}{N_{j}^{2}} I\left(N_{j}>0\right)\right]=e^{-m_{j}} \sum_{k=1}^{\infty} \frac{m^{k}}{k^{2} k !}<e^{-m_{j}}\left(m_{j}+\sum_{k=0}^{\infty} \frac{m_{j}^{k}}{(k+2) !}\right)=$ $m_{j} e^{-m_{j}}+m_{j}^{-2}=O\left(m_{j}^{-2}\right)=o\left(A\left(m_{j}\right)\right) \rightarrow 0$ as $m_{j} \rightarrow \infty$, and $R(k)=$ $\int k^{2}(u) d u$.

Similar arguments yield the following expression for the second summand in expression (A.8)

$$
\operatorname{Var}\left[\mathbb{E}\left[N_{j}^{-2} \sum_{i_{1}=1}^{N_{j}} \sum_{i_{2} \neq i_{1}} k_{G_{j}}\left(\mathbf{x}_{\mathbf{i}_{1}}-\mathbf{x}_{\mathbf{i}_{2}}\right] \mid N_{j}>0\right]\right]=\psi_{j}^{2} O\left(B\left(m_{j}\right)\right)
$$


Plugging expressions (A.10) and (A.11) into expression (A.8) and taking into account that $B\left(m_{j}\right)=o\left(A\left(m_{j}\right)\right)$, the variance of $\psi_{j}$, for $j=1,2$ is

$$
\left.\operatorname{Var} \hat{\psi}_{j}=\left(2 B\left(m_{j}\right)\right)\left|G_{j}\right|^{-1 / 2} \psi_{j} R(k)+4 A\left(m_{j}\right)\left(\int \lambda_{0 j}^{3}(x) d x-\psi_{j}^{2}\right)\right)(1+o(1))
$$

The variance of the cross-product functional $\hat{\psi}_{12}$ is

$$
\begin{aligned}
\mathbb{V a r} \hat{\psi}_{12} & =\mathbb{V} a r\left[\left(N_{1} N_{2}\right)^{-1} \sum_{i=1}^{N_{1}} \sum_{j=N_{1}+1}^{N} k_{G_{1}}\left(\mathbf{x}_{\mathbf{i}}-\mathbf{x}_{\mathbf{j}}\right) I\left(N_{1}>0\right) I\left(N_{2}>0\right)\right] \\
& =\mathbb{E}\left[\mathbb{V} a r\left[\left(N_{1} N_{2}\right)^{-1} \sum_{i=1}^{N_{1}} \sum_{j=N_{1}+1}^{N} k_{G_{1}}\left(\mathbf{x}_{\mathbf{i}}-\mathbf{x}_{\mathbf{j}}\right) \mid N_{1}>0, N_{2}>0\right]\right] \\
& =\mathbb{V} a r\left[\mathbb{E}\left[\left(N_{1} N_{2}\right)^{-1} \sum_{i=1}^{N_{1}} \sum_{j=N_{1}+1}^{N} k_{G_{1}}\left(\mathbf{x}_{\mathbf{i}}-\mathbf{x}_{\mathbf{j}}\right) \mid N_{1}>0, N_{2}>0\right]\right]
\end{aligned}
$$

Theorem 1 in Lee and Dehling (2005) and some Taylor expansions yield the following expression for the variance in the first summand in expression (A.13)

$$
\begin{aligned}
& \operatorname{Var}\left[\left(N_{1} N_{2}\right)^{-1} \sum_{i=1}^{N_{1}} \sum_{j=N_{1}+1}^{N} k_{G_{1}}\left(\mathbf{x}_{\mathbf{i}}-\mathbf{x}_{\mathbf{j}}\right) \mid N_{1}>0, N_{2}>0\right]=N_{1}^{-1} \operatorname{Var}\left[\left(k_{G_{1}} * \lambda_{02}\right)(x)\right] \\
& =N_{1}^{-1} \mathbb{E}\left[\left(k_{G_{1}} * \lambda_{02}\right)^{2}(x)\right]-N_{1}^{-1} \mathbb{E}^{2}\left[\left(k_{G_{1}} * \lambda_{02}\right)(x)\right]
\end{aligned}
$$

where

$$
\begin{aligned}
\mathbb{E}\left[\left(k_{G_{1}} * \lambda_{02}\right)^{2}(x)\right] & =\int\left(\left(k_{G_{1}} * \lambda_{02}\right)(x)\right)^{2} \lambda_{01}(x) d x \\
& =\int\left(\int k_{G_{1}}(x-y) \lambda_{02}(y) d y\right)^{2} \lambda_{01}(x) d x \\
& =\int\left(\lambda_{02}(x)(1+o(1))\right)^{2} \lambda_{01}(x) d x=\int \lambda_{01}(x) \lambda_{02}^{2}(x) d x(1+o(1))
\end{aligned}
$$


and

$$
\begin{aligned}
\mathbb{E}\left[\left(k_{G_{1}} * \lambda_{02}\right)(x)\right] & =\int\left(k_{G_{1}} * \lambda_{02}\right)(x) \lambda_{01}(x) d x \\
& =\iint k_{G_{1}}(x-y) \lambda_{02}(y) \lambda_{01}(x) d y d x \\
& =\int \lambda_{01}(x) \lambda_{02}(x) d x(1+o(1))=\psi_{12}(1+o(1))
\end{aligned}
$$

Plugging these values into expression (A.14), using the properties of measurable functions of a Poisson point process (Cucala, 2006), and taking into account that $\mathbf{X}_{\mathbf{1}}$ and $\mathbf{X}_{2}$ are assumed to be independent, and consequently $N_{1}$ and $N_{2}$ are independent Poisson distributions, we obtain the following expression for the first summand of (A.13)

$$
\begin{aligned}
& \mathbb{E}\left[\operatorname{Var}\left[\left(N_{1} N_{2}\right)^{-1} \sum_{i=1}^{N_{1}} \sum_{j=N_{1}+1}^{N} k_{G_{1}}\left(\mathbf{x}_{\mathbf{i}}-\mathbf{x}_{\mathbf{j}}\right) \mid N_{1}>0, N_{2}>0\right]\right] \\
& =\mathbb{E}\left[N_{1}^{-1}\left(\int \lambda_{01}(x) \lambda_{02}^{2}(x) d x-\psi_{12}\right)(1+o(1)) \mid N_{1}>0, N_{2}>0\right] \\
& =\sum_{l_{1}=1}^{\infty} \frac{1}{N_{1}} \sum_{l_{2}=1}^{\infty}\left(\int \lambda_{01}(x) \lambda_{02}^{2}(x) d x-\psi_{12}\right)(1+o(1)) P\left(N_{1}=l_{1}\right) P\left(N_{2}=l_{2}\right) \\
& =A\left(m_{1}\right)\left(\int \lambda_{01}(x) \lambda_{02}^{2}(x) d x-\psi_{12}^{2}\right)(1+o(1))
\end{aligned}
$$

Similar arguments yield the following expression for the second summand in expression (A.13)

$\operatorname{Var}\left[\mathbb{E}\left[\left(N_{1} N_{2}\right)^{-1} \sum_{i=1}^{N_{1}} \sum_{j=N_{1}+1}^{N} k_{G_{1}}\left(\mathbf{x}_{\mathbf{i}}-\mathbf{x}_{\mathbf{j}}\right) \mid N_{1}>0, N_{2}>0\right]\right]=\psi_{12}^{2} O\left(e^{-m_{1}}+e^{-m_{1}}\right)$

since $O\left(e^{-m_{1}}+e^{-m_{1}}\right)=o\left(A\left(m_{1}\right)\right)$, the first summand is the dominant term in the variance of the cross-product functional $\hat{\psi}_{12}$, thus

$$
\operatorname{Var} \hat{\psi}_{12}=A\left(m_{1}\right)\left(\int \lambda_{01}(x) \lambda_{02}^{2}(x) d x-\psi_{12}^{2}\right)(1+o(1))
$$


and

$$
\operatorname{Var} \hat{\psi}_{21}=A\left(m_{2}\right)\left(\int \lambda_{01}^{2}(x) \lambda_{02}(x) d x-\psi_{21}^{2}\right)(1+o(1))
$$

The law of total covariance yields the following expression for the crosscovariance

$$
\begin{aligned}
\operatorname{Cov}\left(\hat{\psi}_{1}, \hat{\psi}_{12}\right) & =\mathbb{E}\left[\operatorname{Cov}\left(\hat{\psi}_{1}, \hat{\psi}_{12} \mid N_{1}>0, N_{2}>0\right)\right] \\
& +\mathbb{C o v}\left(\mathbb{E}\left[\hat{\psi}_{1} \mid N_{1}>0, N_{2}>0\right], \mathbb{E}\left[\hat{\psi}_{12} \mid N_{1}>0, N_{2}>0\right]\right)
\end{aligned}
$$

The covariance in the first summand of expression (A.20) is

$$
\begin{aligned}
& \operatorname{Cov}\left(\hat{\psi}_{1}, \hat{\psi}_{12} \mid N_{1}>0, N_{2}>0\right) \\
& =\operatorname{Cov}\left(N_{1}^{-2} \sum_{i_{1}=1}^{N_{1}} \sum_{i_{2}=1}^{N_{1}} k_{G_{1}}\left(\mathbf{x}_{\mathbf{i}_{\mathbf{1}}}-\mathbf{x}_{\mathbf{i}_{\mathbf{2}}}\right),\left(N_{1} N_{2}\right)^{-1} \sum_{i=1}^{N_{1}} \sum_{j=N_{1}+1}^{N} k_{G_{1}}\left(\mathbf{x}_{\mathbf{i}}-\mathbf{x}_{\mathbf{j}}\right) \mid N_{1}>0, N_{2}>0\right) \\
& =\mathbb{E}\left[N_{1}^{-1} k_{G_{1}}\left(\mathbf{x}_{\mathbf{1}, \mathbf{1}}-\mathbf{x}_{\mathbf{1}, \mathbf{2}}\right) k_{G_{1}}\left(\mathbf{x}_{\mathbf{1}, \mathbf{1}}-\mathbf{x}_{\mathbf{2}, \mathbf{1}}\right)\right]-\mathbb{E}\left[N_{1}^{-1} k_{G_{1}}\left(\mathbf{x}_{\mathbf{1}, \mathbf{1}}-\mathbf{x}_{\mathbf{1}, \mathbf{2}}\right)\right] \mathbb{E}\left[k_{G_{1}}\left(\mathbf{x}_{\mathbf{1}, \mathbf{1}}-\mathbf{x}_{\mathbf{2}, \mathbf{1}}\right)\right] \\
& =N_{1}^{-1}\left(\iiint k_{G_{1}}(x-y) k_{G_{1}}(x-z) \lambda_{01}(x) \lambda_{01}(y) \lambda_{02}(z) d x d y d z-\psi_{1} \psi_{12}\right) \\
& =N_{1}^{-1}\left(\int \lambda_{01}^{2}(x) \lambda_{02}(x) d x-\psi_{1} \psi_{12}\right)(1+o(1))
\end{aligned}
$$

Therefore, since the marginal processes are Poisson, the first summand in expression (A.20) is

$$
\begin{aligned}
\mathbb{E}\left[\operatorname{Cov}\left(\hat{\psi}_{1}, \hat{\psi}_{12} \mid N_{1}>0, N_{2}>0\right)\right] & =\sum_{l_{1}=1}^{\infty} \frac{1}{N_{1}} \sum_{l_{2}=1}^{\infty}\left(\int \lambda_{01}^{2}(x) \lambda_{02}(x) d x-\psi_{1} \psi_{12}\right) \\
& P\left(N_{1}=l_{1}\right) P\left(N_{2}=l_{2}\right) \\
& =A\left(m_{1}\right)\left(\int \lambda_{01}^{2}(x) \lambda_{02}(x) d x-\psi_{1} \psi_{12}\right)(1+o(1))
\end{aligned}
$$

Using similar arguments, we have that the second summand in expression (A.20) is

$$
\mathbb{C o v}\left(\mathbb{E}\left[\hat{\psi}_{1} \mid N_{1}>0, N_{2}>0\right], \mathbb{E}\left[\hat{\psi}_{12} \mid N_{1}>0, N_{2}>0\right]\right)=0
$$


Therefore

$$
\begin{aligned}
& \operatorname{Cov}\left(\hat{\psi}_{1}, \hat{\psi}_{12}\right)=A\left(m_{1}\right)\left(\int \lambda_{01}^{2}(x) \lambda_{02}(x) d x-\psi_{1} \psi_{12}\right)(1+o(1)) \\
& \operatorname{Cov}\left(\hat{\psi}_{1}, \hat{\psi}_{21}\right)=A\left(m_{1}\right)\left(\int \lambda_{01}^{2}(x) \lambda_{02}(x) d x-\psi_{1} \psi_{21}\right)(1+o(1)) \\
& \mathbb{C o v}\left(\hat{\psi}_{2}, \hat{\psi}_{12}\right)=A\left(m_{2}\right)\left(\int \lambda_{01}(x) \lambda_{02}^{2}(x) d x-\psi_{2} \psi_{12}\right)(1+o(1)) \\
& \mathbb{C o v}\left(\hat{\psi}_{2}, \hat{\psi}_{21}\right)=A\left(m_{2}\right)\left(\int \lambda_{01}(x) \lambda_{02}^{2}(x) d x-\psi_{2} \psi_{21}\right)(1+o(1))
\end{aligned}
$$

Finally

$$
\begin{aligned}
\operatorname{Cov}\left(\hat{\psi}_{12}, \hat{\psi}_{21}\right) & =\mathbb{E}\left[\operatorname{Cov}\left(\hat{\psi}_{12}, \hat{\psi}_{21} \mid N_{1}>0, N_{2}>0\right)\right] \\
& +\operatorname{Cov}\left(\mathbb{E}\left[\hat{\psi}_{12} \mid N_{1}>0, N_{2}>0\right], \mathbb{E}\left[\hat{\psi}_{21} \mid N_{1}>0, N_{2}>0\right]\right)
\end{aligned}
$$

The covariance in the first summand of expression (A.27) is

$$
\begin{aligned}
& \operatorname{Cov}\left(\hat{\psi}_{12}, \hat{\psi}_{21} \mid N_{1}>0, N_{2}>0\right)=\operatorname{Cov}\left(\left(N_{1} N_{2}\right)^{-1} \sum_{i_{1}=1}^{N_{1}} \sum_{i_{2}=N_{1}+1}^{N} k_{G_{1}}\left(\mathbf{x}_{\mathbf{i}_{\mathbf{1}}}-\mathbf{x}_{\mathbf{i}_{\mathbf{2}}}\right),\right. \\
&\left.\left(N_{1} N_{2}\right)^{-1} \sum_{j_{1}=1}^{N_{1}} \sum_{j_{2}=N_{1}+1}^{N} k_{G_{2}}\left(\mathbf{x}_{\mathbf{j}_{\mathbf{1}}}-\mathbf{x}_{\mathbf{j}_{\mathbf{2}}}\right) \mid N_{1}>0, N_{2}>0\right) \\
&=\left(N_{1} N_{2}\right)^{-1} \mathbb{C o v}\left(k_{G_{1}}\left(\mathbf{x}_{\mathbf{1}, \mathbf{1}}-\mathbf{x}_{\mathbf{2}, \mathbf{1}}\right), k_{G_{2}}\left(\mathbf{x}_{\mathbf{1}, \mathbf{1}}-\mathbf{x}_{\mathbf{2}, \mathbf{1}}\right)\right) \\
&+ N_{2}^{-1}\left(1-N_{1}^{-1}\right) \mathbb{C o v}\left(k_{G_{1}}\left(\mathbf{x}_{\mathbf{1}, \mathbf{1}}-\mathbf{x}_{\mathbf{2}, \mathbf{1}}\right), k_{G_{2}}\left(\mathbf{x}_{\mathbf{1}, \mathbf{2}}-\mathbf{x}_{\mathbf{2}, \mathbf{1}}\right)\right) \\
&+ N_{1}^{-1}\left(1-N_{2}^{-1}\right) \mathbb{C o v}\left(k_{G_{1}}\left(\mathbf{x}_{\mathbf{1}, \mathbf{1}}-\mathbf{x}_{\mathbf{2}, \mathbf{1}}\right), k_{G_{2}}\left(\mathbf{x}_{\mathbf{1}, \mathbf{1}}-\mathbf{x}_{\mathbf{2}, \mathbf{2}}\right)\right) \\
&=\left\{\frac{1}{2}\left(N_{1} N_{2}\right)^{-1}\left(\left|G_{1}\right|^{-1 / 2} \psi_{12} \int k(x) k_{G_{2} G_{1}^{-1}}(x) d x+\left|G_{2}\right|^{-1 / 2} \psi_{21} \int k_{G_{1} G_{2}^{-1}}(x) k(x) d x-2 \psi_{12} \psi_{21}\right)\right. \\
&\left.+N_{2}^{-1}\left(\int \lambda_{01}^{2}(x) \lambda_{02}(x) d x-\psi_{12} \psi_{21}\right)+N_{1}^{-1}\left(\int \lambda_{01}(x) \lambda_{02}^{2}(x) d x-\psi_{12} \psi_{21}\right)\right\}(1+o(1))
\end{aligned}
$$


Plugging expression (A.28) into the first summand of (A.27), and taking into account that the marginal processes are Poisson we obtain

$$
\begin{aligned}
\mathbb{E}[ & \left.\operatorname{Cov}\left(\hat{\psi}_{12}, \hat{\psi}_{21} \mid N_{1}>0, N_{2}>0\right)\right]=\sum_{l_{1}=1}^{\infty} \sum_{l_{2}=1}^{\infty}\left\{\frac { 1 } { 2 } ( N _ { 1 } N _ { 2 } ) ^ { - 1 } \left(\left|G_{1}\right|^{-1 / 2} \psi_{12} \int k(x) k_{G_{2} G_{1}^{-1}}(x) d x\right.\right. \\
& \left.+\left|G_{2}\right|^{-1 / 2} \psi_{21} \int k_{G_{1} G_{2}^{-1}}(x) k(x) d x-2 \psi_{12} \psi_{21}\right)+N_{2}^{-1}\left(\int \lambda_{01}^{2}(x) \lambda_{02}(x) d x-\psi_{12} \psi_{21}\right) \\
& \left.+N_{1}^{-1}\left(\int \lambda_{01}(x) \lambda_{02}^{2}(x) d x-\psi_{12} \psi_{21}\right)\right\} P\left(N_{1}=l_{1}\right) P\left(N_{2}=l_{2}\right)(1+o(1)) \\
= & \left\{\frac{A\left(m_{1}\right) A\left(m_{2}\right)}{2}\left(\left|G_{1}\right|^{-1 / 2} \psi_{12} \int k(x) k_{G_{2} G_{1}^{-1}}(x) d x+\left|G_{2}\right|^{-1 / 2} \psi_{21} \int k_{G_{1} G_{2}^{-1}}(x) k(x) d x-2 \psi_{12} \psi_{21}\right)\right. \\
& \left.+A\left(m_{2}\right)\left(\int \lambda_{01}^{2}(x) \lambda_{02}(x) d x-\psi_{12} \psi_{21}\right)+A\left(m_{1}\right)\left(\int \lambda_{01}(x) \lambda_{02}^{2}(x) d x-\psi_{12} \psi_{21}\right)\right\}(1+o(1))
\end{aligned}
$$

Using similar arguments, we have that the second summand in expression (A.27) is

$$
\operatorname{Cov}\left(\mathbb{E}\left[\hat{\psi}_{12} \mid N_{1}>0, N_{2}>0\right], \mathbb{E}\left[\hat{\psi}_{21} \mid N_{1}>0, N_{2}>0\right]\right)=0
$$

Therefore, the cross-covariance (A.27) is

$$
\begin{aligned}
& \operatorname{Cov}\left(\hat{\psi}_{12}, \hat{\psi}_{21}\right)=\left\{\frac { A ( m _ { 1 } ) A ( m _ { 2 } ) } { 2 } \left(\left|G_{1}\right|^{-1 / 2} \psi_{12} \int k(x) k_{G_{2} G_{1}^{-1}}(x) d x\right.\right. \\
& \left.+\left|G_{2}\right|^{-1 / 2} \psi_{21} \int k_{G_{1} G_{2}^{-1}}(x) k(x) d x-2 \psi_{12} \psi_{21}\right)+A\left(m_{2}\right)\left(\int \lambda_{01}^{2}(x) \lambda_{02}(x) d x-\psi_{12} \psi_{21}\right) \\
& \left.+A\left(m_{1}\right)\left(\int \lambda_{01}(x) \lambda_{02}^{2}(x) d x-\psi_{12} \psi_{21}\right)\right\}(1+o(1))
\end{aligned}
$$

Therefore, if we plug expressions (A.12),(A.18), (A.19), (A.23) - (A.26) 
and (A.30) into (A.7) we obtain

$$
\begin{aligned}
& \operatorname{Var} \hat{T}=\left\{2 B\left(m_{1}\right)\left|G_{1}\right|^{-1 / 2} \psi_{1} R(k)+4 A\left(m_{1}\right)\left(\int \lambda_{01}^{3}(x) d x-\psi_{1}^{2}\right)\right. \\
& +2 B\left(m_{2}\right)\left|G_{2}\right|^{-1 / 2} \psi_{2} R(k)+4 A\left(m_{2}\right)\left(\int \lambda_{02}^{3}(x) d x-\psi_{2}^{2}\right) \\
& +3 A\left(m_{1}\right) \int \lambda_{01}(x) \lambda_{02}^{2}(x) d x+3 A\left(m_{2}\right) \int \lambda_{01}^{2}(x) \lambda_{02}(x) d x \\
& -4 A\left(m_{1}\right) \int \lambda_{01}^{2}(x) \lambda_{02}(x) d x-4 A\left(m_{2}\right) \int \lambda_{01}(x) \lambda_{02}^{2}(x) d x \\
& +A\left(m_{1}\right) A\left(m_{2}\right)\left(\left|G_{1}\right|^{-1 / 2} \psi_{12} \int k(x) k_{G_{2} G_{1}^{-1}}(x) d x+\left|G_{2}\right|^{-1 / 2} \psi_{21} \int k_{G_{1} G_{2}^{-1}}(x) k(x) d x\right. \\
& \left.-2 \psi_{12} \psi_{21}\right)-A\left(m_{1}\right) \psi_{12}^{2}-A\left(m_{2}\right) \psi_{21}^{2}+2\left(A\left(m_{1}\right) \psi_{1} \psi_{12}+A\left(m_{1}\right) \psi_{1} \psi_{21}\right. \\
& \left.\left.+A\left(m_{2}\right) \psi_{2} \psi_{12}+A\left(m_{2}\right) \psi_{2} \psi_{21}-A\left(m_{1}\right) \psi_{12} \psi_{21}-A\left(m_{2}\right) \psi_{12} \psi_{21}\right)\right\}(1+o(1))
\end{aligned}
$$

According to Chacón and Duong (2010), for $i, j=1,2$ the optimal bandwidths $G_{j}$ have order $O\left(m^{-1 / 2}\right)$, thus $B\left(m_{j}\right)\left|G_{j}\right|^{-1 / 2}=O\left(m^{-3 / 2}\right)<$ $O\left(A\left(m_{j}\right)\right)$ and $A\left(m_{i}\right) A\left(m_{j}\right)\left|G_{j}\right|^{1 / 2}<O\left(A\left(m_{i}\right)\right)$. Therefore the dominant terms in expression (A.31) are those of order $A\left(m_{j}\right)$. In addition, under the null hypothesis $\lambda_{01}(\cdot)=\lambda_{02}(\cdot)=\lambda_{0}(\cdot)$ and, consequently $\psi_{1}=\psi_{2}=\psi_{21}=$ $\psi_{21}=\psi$. Thus, the null variance of $\hat{T}$ reduces to expression 6

$$
\operatorname{Var} \hat{T}=3\left(A\left(m_{1}\right)+A\left(m_{2}\right)\right)\left(\int \lambda_{0}^{3}(x) d x-\psi^{2}\right)(1+o(1))
$$

\section{Asymptotic normality}

The Central Limit Theorem for U-statistics of Poisson point processes (see Theorem 4.7 in Reitzner et al. (2013)) asserts the asymptotic normality of $\psi_{j}$ for $j=1,2$.

In order to prove the asymptotic normality of $\psi_{i j}, i, j=1,2$, we apply a Hoeffding's decomposition (Lee and Dehling, 2005) to $\phi\left(\mathbf{x}_{\mathbf{i}, \mathbf{1}}, \mathbf{x}_{\mathbf{j}, \mathbf{1}}\right)=$ $k_{G_{j}}\left(\mathbf{x}_{\mathbf{i}, \mathbf{1}}-\mathbf{x}_{\mathbf{j}, \mathbf{1}}\right)$ for $j=1,2$, which yields

$$
k_{G_{j}}\left(\mathbf{x}_{\mathbf{i}, \mathbf{1}}-\mathbf{x}_{\mathbf{j}, \mathbf{1}}\right)=\theta+k_{1, G_{j}}\left(\mathbf{x}_{\mathbf{i}, \mathbf{1}}\right)+k_{2, G_{j}}\left(\mathbf{x}_{\mathbf{j}, \mathbf{1}}\right)+k_{G_{j}}^{*}\left(\mathbf{x}_{\mathbf{i}, \mathbf{1}}-\mathbf{x}_{\mathbf{j}, \mathbf{2}}\right)
$$


where $\theta=E k_{G_{j}}\left(\mathbf{x}_{\mathbf{i}, \mathbf{1}}-\mathbf{x}_{\mathbf{j}, \mathbf{1}}\right)$ and

$$
\begin{aligned}
& k_{1, G_{j}}(x)=\mathbb{E}_{\mathbf{X}_{\mathbf{2}}}\left[k_{G_{j}}\left(x-\mathbf{x}_{\mathbf{j}, \mathbf{2}}\right)\right]-\theta=\int k_{G_{j}}(x-y) \lambda_{02}(y) d y-\theta \\
& k_{2, G_{j}}(y)=\mathbb{E}_{\mathbf{X}_{\mathbf{i}, \mathbf{1}}}\left[k_{G_{j}}\left(\mathbf{x}_{\mathbf{1}}-y\right)\right]-\theta=\int k_{G_{j}}(x-y) \lambda_{01}(x) d x-\theta \\
& k_{G_{j}}^{*}\left(\mathbf{x}_{\mathbf{i}, \mathbf{1}}-\mathbf{x}_{\mathbf{j}, \mathbf{2}}\right)=k_{G_{j}}\left(\mathbf{x}_{\mathbf{i}, \mathbf{1}}-\mathbf{x}_{\mathbf{j}, \mathbf{1}}\right)-k_{1, G_{j}}\left(\mathbf{x}_{\mathbf{i}, \mathbf{1}}\right)-k_{2, G_{j}}\left(\mathbf{x}_{\mathbf{j}, \mathbf{1}}\right)+\theta
\end{aligned}
$$

Therefore

$$
\begin{aligned}
& \hat{\psi}_{12}=\left(N_{1} N_{2}\right)^{-1} \sum_{i=1}^{N_{1}} \sum_{j=N_{1}+1}^{N} k_{G_{1}}\left(\mathbf{x}_{\mathbf{i}}-\mathbf{x}_{\mathbf{j}}\right) I\left(N_{1}>0\right) I\left(N_{2}>0\right) \\
& =N_{1}^{-1} \sum_{i=1}^{N_{1}}\left(K_{G_{1}} * \lambda_{02}\right)\left(\mathbf{x}_{1}\right) I\left(N_{1}>0\right)+N_{2}^{-1} \sum_{i=1}^{N_{2}}\left(K_{G_{2}} * \lambda_{01}\right)\left(\mathbf{x}_{\mathbf{2}}\right) I\left(N_{2}>0\right) \\
& +\left(N_{1} N_{2}\right)^{-1} \sum_{i=1}^{N_{1}} \sum_{j=N_{1}+1}^{N} k_{G_{1}}^{*}\left(\mathbf{x}_{\mathbf{i}}-\mathbf{x}_{\mathbf{j}}\right) I\left(N_{1}>0\right) I\left(N_{2}>0\right)
\end{aligned}
$$

Since $O\left(N_{1} N_{2}\right)<O\left(N_{i}\right), i=1,2$, the two first summands are the dominant terms in expression (A.32). These terms are U-statistics of spatial Poisson pint processes, thus asymptotically normal according to Theorem 4.7 in Reitzner et al. (2013). Therefore, $\hat{T}=\hat{\psi}_{1}+\hat{\psi}_{2}-\left(\hat{\psi}_{12}+\hat{\psi}_{21}\right)$ is asymptotically normal as stated in expression (4).

\section{Acknowledgements}

The authors acknowledge the anonymous reviewers and the Associate Editor for their valuable comments that substantially improved this paper. They also acknowledge the support of the Project MTM2008-0310 from the Spanish Ministry of Science and Innovation, Projects MTM2010-14961, MTM2013-41383-P, MTM2013-43917-P, MTM2016-78917-R and MTM201676969-P from the Spanish Ministry of Economy and Competitivity, grant P1-1B2015-60 from Bancaja Foundation, and IAP network StUDyS from the Belgian Science Policy. 


\section{References}

Alba-Fernández, M., Ariza-López, F., Jiménez-Gamero, M. D., RodríguezAvi, J., 2016. On the similarity analysis of spatial patterns. Spatial Statistics, (In press, available online: 18 August 2016).

doi: $10.1016 / \mathrm{j}$.spasta.2016.07.004

Andresen, M. A., 2009. Testing for similarity in area-based spatial patterns: a nonparametric Monte Carlo approach. Appl. Geogr 29 (3), 333-345.

doi: $10.1016 / j$.apgeog. 2008.12.004

Baddeley, A., Turner, R., 2005. Spatstat: an R package for analyzing spatial point patterns. J Stat Sofw 12 (6), 1-42.

doi: $10.18637 /$ jss.v012.i06

Boubeta, M., Lombardía, M. J., Morales, D., 2015. Empirical best prediction under area-level Poisson mixed models. Test. 25 (3), 548-569.

doi: $10.1007 / \mathrm{s} 11749-015-0469-8$

Chacón, J. E., Duong, T., 2010. Multivariate plug-in bandwidth selection with unconstrained pilot bandwidth matrices. Test 19 (2), 375-398.

doi: $10.1007 / \mathrm{s} 11749-009-0168-4$

Consellería do Medio Rural, 2016. Plan de prevención e defensa contra os incendios forestais de Galicia. PLADIGA (http : //mediorural.xunta.gal/es/areas/forestal/incendios orestales/pladiga $_{2} 016 /$ ).

Cucala, L., 2006. Espacements bidimensionnels et données entachées d'erreurs dans l'analyse des processus ponctuels spatiaux. Ph.D. thesis, Université des Sciences Sociales, Toulouse I.

Davies, T. M., Hazelton, M. L., 2010. Adaptive kernel estimation of spatial relative risk. Stat Med 29 (23), 2423-2437.

doi: $10.1002 / \mathrm{sim} .3995$

Díaz-Avalos, C., Juan, P., Mateu, J., 2013. Similarity measures of conditional intensity functions to test separability in multidimensional point processes. Stoch Env Res Risk A 27 (5), 1193-1205.

doi: $10.1007 / \mathrm{s} 00477-012-0654-1$

Diggle, P. J., 2013. Statistical Analysis of Spatial and Spatio-Temporal Point Patterns, 3rd Edition. CRC Press, Boca Raton, Florida. 
Duong, T., 2013a. ks: Kernel smoothing. R package version 1.8.13. url: http://CRAN.R-project.org/package=ks.

Duong, T., 2013b. Local significant differences from nonparametric twosample tests. J. Nonparametric Stat. 25 (3), 635-645.

doi: $10.1080 / 10485252.2013 .810217$

Duong, T., Goud, B., Schauer, K., 2012. Closed-form density-based framework for automatic detection of cellular morphology changes. Proc. Natl. Acad. Sci. U.S.A. 109 (22), 8382-8387.

doi: $10.1073 /$ pnas. 1117796109

Fibich, P., Lep, J., Novotn, V., Klime, P., Titel, J., Molem, K., Damas, K., Weiblen, G. D., 2016. Spatial patterns of tree species distribution in New Guinea primary and secondary lowland rain forest. J. Veg. Sci.(In press, available online 11 January 2016).

doi: $10.1111 /$ jvs. 12363

Fuentes-Santos, I., González-Manteiga, W., Mateu, J., 2016. Consistent smooth bootstrap kernel intensity estimation for inhomogeneous spatial Poisson point processes. Scand. J. Stat. 43 (2), 416-435.

doi: $10.1111 /$ sjos. 12183

Fuentes-Santos, I., Marey-Pérez, M., González-Manteiga, W., 2013. Forest fire spatial pattern analysis in Galicia (NW Spain). J. Environ. Manage. $128,30-42$.

doi: $10.1016 / j \cdot j$ envman.2013.04.020

Getzin, S., Wiegand, T., Wiegand, K., He, F., 2008. Heterogeneity influences spatial patterns and demographics in forest stands. J. Ecol. 96 (4), 807820.

doi: $10.1111 / \mathrm{j} .1365-2745.2008 .01377 . x$

Hahn, U., 2012. A studentized permutation test for the comparison of spatial point patterns. J. Am. Stat. Assoc. 107 (498), 754-764.

doi: $10.1080 / 01621459.2012 .688463$

Hering, A. S., Bell, C. L., Genton, M. G., 2009. Modeling spatio-temporal wildfire ignition point patterns. Environ. Ecol. Stat 16 (2), 225-250. doi: $10.1007 /$ s10651-007-0080-6 
Illian, D. J., Penttinen, P. A., Stoyan, D. H., Stoyan, D., 2008. Statistical Analysis and Modelling of Spatial Point Patterns. John Wiley \& Sons, Chichester, UK.

Juan, P., Mateu, J., Saez, M., 2012. Pinpointing spatio-temporal interactions in wildfire patterns. Stoch Env Res Risk A 26 (8), 1131-1150.

doi: $10.1007 / \mathrm{s} 00477-012-0568-y$

Kelsall, J. E., Diggle, P. J., 1995. Non-parametric estimation of spatial variation in relative risk. Stat Med 14, 2335-2342.

doi: $10.1002 / \operatorname{sim} .4780142106$

Lee, M.-L. T., Dehling, H. G., 2005. Generalized two-sample U-statistics for clustered data. Statistica Neerlandica 59 (3), 313-323.

doi: $10.1111 / \mathrm{j} .1467-9574.2005 .00298 . \mathrm{x}$

Perry, G. L., Miller, B. P., Enright, N. J., 2006. A comparison of methods for the statistical analysis of spatial point patterns in plant ecology. Plant Ecol. 187 (1), 59-82.

doi: $10.1007 / \mathrm{s} 11258-006-9133-4$

R Core Team, 2014. R: A Language and Environment for Statistical Computing. R Foundation for Statistical Computing, Vienna, Austria, url: http://www.R-project.org/.

Reitzner, M., Schulte, M., others, 2013. Central limit theorems for Ustatistics of Poisson point processes. Ann Probab 41 (6), 3879-3909.

doi: $10.1214 / 12-A 0 P 817$

Ríos-Pena, L., Cadarso-Suárez, C., Kneib, T., Marey-Pérez, M., 2015. Applying Binary Structured Adiditive Regression (STAR) for Predicting Wildfire in Galicia, Spain. Procedia Environmental Sciences 27, 123-126.

doi: $10.1016 / \mathrm{j} \cdot$ proenv .2015 .07 .121

Ripley, B., 1977. Modelling spatial patterns (with discussion). J. R. Stat. Soc B 39, 172-212.

Ripley, B., 1981. Spatial Statistics. Wiley, New York.

Schoenberg, F. P., Jun. 2004. Testing separability in spatial-temporal marked point processes. Biometrics 60 (2), 471-481.

doi: $10.1111 / \mathrm{j} .0006-341 \mathrm{X} .2004 .00192 . \mathrm{x}$ 
Serra, L., Saez, M., Juan, P., Varga, D., Mateu, J., 2014. A spatio-temporal Poisson hurdle point process to model wildfires. Stoch Env Res Risk A 28 (7), 1671-1684.

doi: $10.1007 / \mathrm{s} 00477-013-0823-\mathrm{x}$

Wand, M. P., Jones, M. C., Dec. 1995. Kernel Smoothing. CRC Press, London. 